\title{
DINÂMICA DO SUB-BOSQUE E DO ESTADO ARBÓREO DE FLORSTA TROPICAL PRIMÁRIA FRAGMENTADA NA AMAZÔNIA ORIENTAL'
}

\author{
Rafael de Paiva SALOMÃO², Aires Henriques de MATOS ${ }^{3}$, Nélson de Araújo ROSA ${ }^{2}$
}

RESUMO - Objetivou-se neste trabalho avaliar a dinâmica florestal, sobretudo da regcneraçào natural, em um fragmento de floresta tropical primária, entre 1998 e 1999, em Pcixe-Boi (PA). Foram demarcadas très parcelas pennanentes ( 1 ha cada) onde todos os indivíduos com $\mathrm{DAP} \geq 10 \mathrm{~cm}$ foram registrados; os individuos com $10 \mathrm{~cm}>$ DAP $\geq 5 \mathrm{~cm}$ foram amostrados em $6.000 \mathrm{~m}^{2}$, aqueles cntre $5 \mathrm{~cm}>$ DAP $\geq 2 \mathrm{~cm}$ em $2.400 \mathrm{~m}^{2} \mathrm{e}$ com DAP $<2 \mathrm{~cm}$ em $240 \mathrm{~m}^{2}$. Foram estimados 143.000 indivíduos, desde plântulas até árvores pertencentes a 337 espécies $\mathrm{c} 76$ familias. Mimosaceac foi a família de maior riqueza (44 espécies); 14 familias ocorreram com uma única espécic sendo que metade delas apresentaram também um único individuo. Independentementc da classe diamétrica verificou-se o egresso de 56 espécies versus o ingresso de 68, gerando um ganho liquido

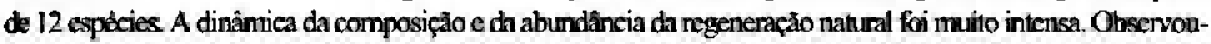
se a saida de uma familia face ao ingesso de outms 14 , aumentando em quase $30 \%$ o nimero de spicies. A maior mortalidade foi verificada em Bauhinia cf. rutilans e Mabea aff. speciosa (300 e 21 individuos). Rinorea negleta e Lecythis idatimon recrutaram 171 e 89 espécimes. A razão recrutanento/mortalidade foi, em quaisquer das classes diamétricas, sempre superior a unidade. O estoque de mudas para sc obter uma árvore, uma arvoreta e uma vara foi, respectivamente de 297,160 e 48 mudas. O númcro de espécies e a abundância aumentaram no periodo, assim como a área basal e a biomassa.

Palavras-chave: Dinâmica florestal, Estrutura, Composição floristica, Floresta tropical primária, Sub-bosque, Amazônia

\section{Dynamics of Undergrowth and Arboreal Stratum in a Fragment of Primary Tropical Forest in Eastern Amazonia.}

ABSTRACT - The prescnt paper aims at evaluating the forest dynamics - mainly that brought by natural regenteration -in a fragnent of dense, verna firme tropical primary fores fron 1908 though 1999 , municipality of Peixe-Boi (PA). Three 1 ha permanent plots were marked off and every individual within having $\mathrm{DBH} \geq 10 \mathrm{~cm}$ was registered. Individuals having $\mathrm{DBH}$ between 5 and $10 \mathrm{~cm}$ were sampled out in an area of $6.000 \mathrm{~m}^{2}$, those having DBH between 2 and $5 \mathrm{~cm}$ were sampled out in an area totaling 2.400 $\mathrm{m}^{2}$, and individuals having DBH under $2 \mathrm{~cm}$ were sampled in $240 \mathrm{~m}^{2}$. There are about 143.000 individeals ranging from seedlings to trees, of 337 species and 76 families. Mimosaceae was the family with higher diversity (44 species). Fourtecn families occurred as an only species, including 7 families occurring also as an only individual. Considering the whole sample (that is, independenty fom damotric assis) the ogression of 56 species versus ingression of 68 was verified, producing a net gain of 12 species. The dynamics of species richeness in natural regeneration is remarkably intense, and the dynamics of abundance of natural regeneration, is more intense than that of floristic richeness. It was also observed that only onc family left, while 14 other families cntered, thus enriching biodiversity in almost $30 \%$. The highest mortality verified was of Bauhinia cf. rutilans and Mabea aff.speciosa (300 and 21 individuals). Rinorea negleta and Lecythis idatimon displayed the recruitment of 171 and 89 specimens. The recruitment/mortality rate was higher than one unit in all diametric classes. The proportion of seedlings needed to obtain a trec, a sapling and a stemlet is 297,160 e 48 respectively. Richeness and abundance increased during the one year period, as did basal area and biomass.

Key-words: Forest dynamics, Primary tropical forest, Undergrowth, Diversity and structure, Amazon.

'Trabalho financiado com recursos do Convênio MMA/FNMA № 041/97 e CNPq/MPEG Projeto Interaçøes de Floreats.

${ }^{2}$ MCT/Museu Paraense Emilio Gocldi, Dept ${ }^{\circ}$ de Botânica, C.P. 399, 66040-170, Belèm, Pará. E-mail: alomao@muscu-goeldi.br

${ }^{3}$ Bolstsa PCI/MCT-MPEG, Dept de Botânica. 


\section{Introdução}

$\mathrm{O}$ conhecimento da dinâmica de uma floresta permite avaliar, entre outros, o crescimento, a mortalidade e o recrutamento possibilitando também ter-se noção das variações da composição florística a nível de famílias, gêneros e/ou espécies e se essas variações são cíclicas ou não e, se o são, como se comportam estes ciclos. Estes conhecimentos são de extrema importância para o manejo sustentável do recurso florestal e para a preservação dos diversos ecossistemas florestais e da diversidade biológica a eles intrínseca.

A diversidade de florestas tropicais foi correlacionada por Phillips et al. (1994) com a dinâmica da mortalidade e recrutamento. Analisando mais de duas dezenas de florestas primárias nas regiões tropicais do mundo, demonstraram que a média entre a taxa de mortalidade e recrutamento é, entre outras variáveis, a que melhor explica as diferenças de riquezas das amostras, estabeleceram, então, uma correlação positiva entre a dinâmica e a riqueza das florestas.

Uhl (1982), analisando as florestas de terra firme da Amazônia venezuelana, sugere que a dinâmica de diferentes comunidades seja similar, visto que alguns estudos mostraram grande semelhança nas taxas de mortalidade dessas distintas comunidades. Por outro lado, Carey et al. (1994) demonstram que no mesmo tipo de vegetação, a taxa de mortalidade é muito variável entre diferentes parcelas e diferentes anos de monitoramento, o que permitiu deduzir que a taxa é aleatória entre parcelas não estando relacionada com a abundância ou a biomassa amostradas no ano inicial.

Fatores climáticos como a pluviosidade e sazonalidade e edáficos como a fertilidade, influenciam diretamente a diversidade de árvores e cipós das florestas neotropicais, como demonstraram Clinebell II et. al. (1995). Oliveira (1997) analisou a diversidade, a estrutura e a dinâmica da floresta de terra firme da região de Manaus, correlacionando os principais fatores usualmente relacionados à alta diversidade de árvores em florestas tropicais - pluviosidade, sazonalidade, qualidade do solo e dinâmica da mortalidade de árvores. Observou que os resultados obtidos para a região de Manaus estão em desacordo com a postulada correlação positiva entre a taxa de mortalidade e a diversidade de árvores, encontrada em vários estudos de florestas tropicais de todo o mundo. Concluiu que as florestas de Manaus estão entre as mais ricas em espécies mas entre as menos dinâmicas, segundo a taxa de mortalidade.

Ainda hoje existe um número reduzido de estudos de crescimento, mortalidade e dinâmica de florestas neotropicais. Alguns outros com significativa relevância são os de Pires \& Moraes (1966), Moraes (1970), Pires (1976), Veillon et al. (1976), Putz \& Milton (1982), Salomão et al. (1988), Jardim (1990), Rankin-de- 
Merona et al. (1990, 1992), Salomão (1991), Phillips \& Gentry (1994), Felfili, J.M. (1995), Condit et al. (1996), Carvalho (1999) e Carvalho et al. (1999). Objetiva-se neste trabalho avaliar as modificações anuais da estrutura e da composição florística da regeneração natural e, também, do estrato arbóreo de um fragmento de floresta tropical primária, no período compreendido entre 1998 e 1999 , localizada na Amazônia oriental, nordeste do Estado do Pará.

\section{Metodọlogia}

\section{Área de Estudo}

As unidades amostrais (ou parcelas permanentes) foram implantadas num fragmento de floresta tropical primária, c.a. 200 ha, situado no município de Peixe-Boi $\left(1^{\circ}\right.$ $\left.11^{\prime} \mathrm{S} / 47^{\circ} 19^{\prime} \mathrm{W}\right)$, na parte central da microrregião Bragantina, nordeste do Estado do Pará. Esta é a mais antiga área de colonização agrícola de toda a Amazônia brasileira, com mais de $98 \%$ de alteração da cobertura florestal original, já em 1986, ocorrendo àquela época, tão somente $9.400 \mathrm{~km}^{2}$ de vegetação primária (IBDF/SUDAM, 1988).

O clima da região é quente e úmido (três meses secos), temperatura média oscilando entre $24^{\circ}-26^{\circ} \mathrm{C}$, precipitação média anual variando entre $2.000-3.000$ $\mathrm{mm}$ (entre setembro e novembro, meses mais secos e mais quentes, a precipitação mensal oscila entre 50 a $100 \mathrm{~mm}$ ) e média anual de insolação flutuando entre $2.200-2.400$ horas; a umidade relativa anual raramente é inferior a $80 \%$
(Diniz, 1986).

\section{Caracterização das Unidades Amostrais}

Em locais previamente selecionados do fragmento florestal foram alocadas três unidades amostrais (UA), de um hectare cada $(500 \mathrm{~m} \times 20 \mathrm{~m}$, subdividido em 40 parcelas de $25 \mathrm{~m} \times 10 \mathrm{~m}$ ), para estudos da vegetação. Todas as parcelas foram numeradas e identificadas com placas de alumínio. Em cada UA (1 ha), temse 40 parcelas, e 8 subparcelas para cada sub-amostragem: S1, S2 e S3 (Tab. 1). A definição da localização dessas oito subparcelas foi aleatória (sorteadas entre as 40 parcelas) e demarcada sempre a partir do início da respectiva parcela. A intensidade de amostragem (20\%) das subparcelas foi pré-estabelecida em função dos custos e tempo disponíveis para a execução dos trabalhos.

\section{Procedimentos de Medição}

A medição do diâmetro obtida através de fita diamétrica ou paquímetro Usou-se uma vara de alumínio com $1,30 \mathrm{~m}$ de comprimento para determinação do local exato de medição do DAP, que era então fixado no fuste com Marcador Esferográfico Riskey ${ }^{\circledR}$ ou com lápis de cêra próprio para madeira. As placas de alumínio foram fixadas nos indivíduos com arame galvanizado que os circundavam com uma folga, a mais ou menos $1,50 \mathrm{~m}$ acima do solo. As árvores mortas e em pé (DAP $\geq 10$ $\mathrm{cm}$ ) foram anotadas (mas não registradas) para se avaliar a 
Tabela 1. Esquema da amostragem da vegetação em cada Unidade Amostral permanente (UA), de 1 ha cada, no fragmento de floresta tropical primária densa, Municipio de Peixe-Boi, Pará. PARCELAS / SUBPARCELAS

\begin{tabular}{|c|c|c|c|c|c|c|}
\hline \multirow[b]{2}{*}{$\begin{array}{l}\text { CATEGORIA DE } \\
\text { AMOSTRAGEM }\end{array}$} & \multirow[b]{2}{*}{ SIGLA } & & \multirow[b]{2}{*}{ COA DA PLACA ${ }^{(0)}$} & \multirow[b]{2}{*}{$N^{\circ}$ DE REGISTRO NA PLACA } & \multirow[b]{2}{*}{ INDIVIDUO QUALIFICADO'] } \\
\hline & & QUANTIDADE & DIMENSÃO & & & \\
\hline Amostragem Parcela & AP & $40^{(4)}$ & $\begin{array}{l}25 \mathrm{~m} \times 10 \mathrm{~m} \\
(0,025 \mathrm{ha})\end{array}$ & Aluminio & $<n^{0} \cup A>-<n o$ parc $><n^{0}$ ind $>$ & $\mathrm{DAP} \geq 10,0 \mathrm{~cm}$ \\
\hline Sub-amostragem 1 & S1 & 8 (5) & $\begin{array}{l}25 \mathrm{~m} \times 10 \mathrm{~m} \\
(0,025 \mathrm{ha})\end{array}$ & Amarela & $<n^{0} \cup A>-<n o$ parc. $><n^{0}$ ind. $>$ & $10,0 \mathrm{~cm}>$ DAP $\geq 5.0 \mathrm{~cm}$ \\
\hline Sub-amostragem 2 & S2 & $8^{(i)}$ & $\begin{array}{l}10 \mathrm{~m} \times 10 \mathrm{~m} \\
(0,010 \mathrm{ha})\end{array}$ & Laranja & $\left\langle n^{0} U A\right\rangle-<n o$ parc. $\rangle<n^{0}$ ind. $\rangle$ & $5,0 \mathrm{~cm}>\mathrm{DAP} \geq 2,0 \mathrm{~cm}$ \\
\hline Sub-amostragem 3 & S3 & $8^{(n)}$ & $\begin{array}{l}10 \mathrm{~m} \times 1 \mathrm{~m} \\
(0.001 \mathrm{ha}) \\
\end{array}$ & & Bandeiras de 2 cores & DAP $<2,0 \mathrm{~cm}$ \\
\hline
\end{tabular}

Notas: (1) As placas de alumínio $(7,5 \mathrm{~cm} \times 2,0 \mathrm{~cm}$ previamente perfuradas) usadas em $\mathrm{S} 1$ e $\mathrm{S} 2$ foram pintadas com esmalte sintético spray amarelo e laranja, respectivamente. (2) Em cada UA e para cada tratamento os registros iniciaram-se em 1 e terminaram em $n$ (Ano 1); no Ano 2 iniciaram com $n+1$. (3) Árvore, palmeira, cipó, arbusto, arvoreta ou muda vivos e, também, árvores mortos e em pé, que só eram medidos - não recebiam número de registro nem placa de alumínio. (4) 40 parcelas. (5) 8 subparcelas S1. (6) 8 subparcelas S2. (7) 8 subparcelas S3.

mortalidade. As árvores que se situavam na margem esquerda da UA (parcialmente dentro da UA e parcialmente fora) foram registradas; as da direita não o foram. Pretende-se efetuar medições bianuais nas parcelas e subparcelas.

Para as estimativas das alturas do fuste e da copa, utilizou-se uma vara de $5 \mathrm{~m}$ de comprimento, subdividida de metro em metro. Estas estimativas eram fornecidas sempre pelo menos auxiliar de campo.

\section{Procedimentos de Campo}

Os procedimentos de campo das atividades inerentes às amostragens da vegetação são na descritos na Tabela 2.

\section{Parâmetros Calculados}

A composição florística do fragmento foi caracterizada através das famílias e das espécies presentes nas unidades amostrais. A análise do grau de semelhança entre as UA's foi calculado através do índice de similaridade Sörensen (1943) e a diversidade florística foi analisada através do índice de Shannon \& Wiener (Shannon e Wiener, 1949; Margalef, 1968; Ricklefs, 1979).

As variáveis fitossociológicas como densidade, riqueza, frequência, dominância e indice de valor de importância das espécies e famílias foram calculados de acordo com Cottam \& Curtis (1956) e MuellerDombois \& Ellenberg (1974).

Também foram calculados o número de indivíduos por hectare, a área basal, o volume de madeira (fator de forma $=0,7$ ) e a biomassa aérea viva de acordo com Brown et al. $(1989) \rightarrow \mathrm{Y}=0,0899 *\left(\mathrm{D}^{2} * \mathrm{H} *\right.$ $\mathrm{S})^{0.9522}$; onde: $\mathrm{Y}=$ biomassa aérea em peso seco (kg/árvore); $\mathrm{D}=$ diâmetro (DAP) da árvore $(\mathrm{cm}) ; \mathrm{H}=$ altura total da árvore $(\mathrm{m}) ; \mathrm{S}=$ densidade da madeira $\left.\left(\mathrm{g} / \mathrm{cm}^{3}\right)\right]$. 
Daquelas espécies arbóreas de maior relevância descreveu-se a utilidade mais comum: valor comercial da madeira, assim como o seu uso alternativo, a produção de frutos para o homem e/ou fauna, resina, látex, essência aromática, corante e uso medicinal.

\section{Convenções e Conceituações}

Unidade amostral (UA) tem como sinônimos parcela permanente e unidade amostral permanente; subparcelas são aquelas áreas onde foram avaliados os indivíduos com DAP inferior a $10 \mathrm{~cm}$. Parcelas são as subdivisões, em $\mathrm{n}^{\circ}$ de 40 , da UA onde foram amostrados os indivíduos com $\mathrm{DAP} \geq 10 \mathrm{~cm}$.

Uma premissa adotada neste trabalho refere-se à estratificação vertical do ecossistema florestal: considera-se floresta aquela formação vegetal cujos espécimes tềm DAP $\geq$ $10 \mathrm{~cm}$ e como sub-bosque aquela formação cujos indivíduos estão abaixo deste limite e acima de $2 \mathrm{~cm}$ de DAP (sub-amostragens S1 e S2); a regeneração natural lato sensu seria então aquela formada pelos indivíduos com DAP inferior a $2,0 \mathrm{~cm}(\mathrm{~S} 3)$.

$\mathrm{Na}$ distribuição das famílias, espécies e indivíduos, em classes de variação quantitativa, foi a adotada a seguinte convenção, tendo como referência o Ano 1(1998) e Ano 2 (1999): $\uparrow=$ variável ( $\mathrm{n}^{\circ}$ de famílias, $\mathrm{n}^{\circ}$ de espécies ou $\mathrm{n}^{\mathrm{o}}$ de indivíduos) em crescimento; $\downarrow=$ variável em declínio e, $\mathrm{K}=$ variável constante.

Registro: taxon (unidade taxonômica: família, gênero e espécie) que foi registrado necessariamente no ano 1 (1998).

Ingresso: taxon que entrou na amostragem após o ano 1 .

Tabela 2. Atividades de campo desenvolvidas a para amostragem da vegetação nas parcelas permanentes do fragmento de floresta tropical primária densa, Município de Peixe-Boi, Pará.

\begin{tabular}{ll}
\multicolumn{1}{c}{ ATIVIDADE } & APLICAÇÃO \\
\hline - Numeração das placas: 1 a n (Ano 1) e n+1 a n+... (Ano 2)(1) & AP, S1, S2 \\
- Uso de bandeiras coloridas & $\mathrm{S} 3$ \\
- Identificação e coleta de material para herborização & $\mathrm{AP}, \mathrm{S} 1, \mathrm{~S} 2$ \\
- Coleta de material para xiloteca (2) & $\mathrm{AP}$ \\
- Medição do DAP & $\mathrm{AP}, \mathrm{S} 1, \mathrm{~S} 2$ \\
- Estimação das alturas do fuste e da copa ${ }^{(3)}$ & $\mathrm{AP}$ \\
- Estimação da altura total (3) & $\mathrm{S} 1$ e S2 \\
- Identificação e contagem do número de indivíduos & $\mathrm{S} 3$ \\
\hline
\end{tabular}

Notas: (1) Se no Ano 1 (1998) na Unidade Amostral permanente 1 (UA1) a amostragem da parcela $(\mathrm{AP}=$ indivíduos com DAP ${ }^{3} 10 \mathrm{~cm}$ ) foi de 1 a 480 na $2^{\mathrm{a}}$ mediçāo (Ano 2: 1999) os indivíduos recrutados começaram com o número 481 , semelhantemente para S1 e S2.

(2) Material proveniente de galhos grossos.

(3) Vara de cinco metros subdividida de metro em metro.

(4) AP, S1, S2 e S3: vide Tabela 1. 
Egresso: taxon que saiu da amostragem através da morte do individuo que o representava.

Recrutamento: individuo que atingindo a qualificação diamétrica adotada é registrado a partir do ano 1 .

Mortalidade: refere-se àquele indivíduo que morreu no período considerado.

Perda líquida: é o valor apurado do total no ano inicial, abatido do somatório dos ingressos + egressos.

Ganho líquido: é o valor apurado do total no ano inicial, abatido do somatório dos ingressos + egressos.

\section{Resultados e Discussão}

Os resultados referem-se às duas medições (intervalo de 12 meses) realizadas na amostragem e nas subamostragens S1, S2 e S3.

\section{Florística}

Foram registrados um total de 3.515 indivíduos, 471 espécies e 77 famílias, englobando as duas medições nas três unidades amostrais de monitoramento (Tab. 3). A relação de todas as espécies registradas é apresentada no Anexo 1.

Considerando-se os individuos com DAP $\geq 10 \mathrm{~cm}$ observou-se que a média entre as duas medições foi de 150 espécies $*$ ha $^{-1}$ e de 44 familias $* \mathrm{ha}^{-1}$. Como estes valores refletem a média das três parcelas alocadas em locais distintos do fragmento florestal, procedeu-se ao cálculo do índice de similaridade (S) entre as unidades amostrais, bem como do índice de diversidade $(\mathrm{H})$ - Tabela 4. Para o cálculo foram utilizados os dados do segundo ano de monitoramento.

Existem vários critérios para quantificar a similaridade ou dissimilaridade entre comunidades biológicas de duas áreas. Goodall (1973) propôs que as propriedades do indice têm importância prática, já que seu valor é estimado a partir de amostras. Um bom índice deve ter alta precisão na estimativa de similaridade entre comunidades e baixo potencial de viezes.

Para análise da similaridade

Tabela 3. Totais de indivíduos, espécies e famílias observadas em dois inventários anuais consecutivos, nas 3 unidades amostrais permanentes da floresta tropical primária, nas diversas classes de DAP; Município de Peixe-Boi, Pará.

\begin{tabular}{|c|c|c|c|c|c|c|}
\hline \multirow{2}{*}{ AMOSTRAGEM / CLASSE DIAMETRICA } & \multicolumn{3}{|c|}{ PARCELAS E SUB-PARCELAS } & \multicolumn{3}{|c|}{ TOTAL MONITORADO } \\
\hline & QUANT & ÁREA $\left(m^{2}\right)$ & $\begin{array}{c}\text { AिRA } \\
\text { TOTAL } \\
\left(\mathrm{m}^{2}\right)\end{array}$ & $\begin{array}{c}N^{\circ} D E \\
\text { INDIVIDUOS }\end{array}$ & $\begin{array}{c}\mathrm{N}^{0} \mathrm{DE} \\
\text { ESPECCIES }\end{array}$ & $\begin{array}{l}N^{0} D E \\
\text { FAMILIAS }\end{array}$ \\
\hline Amostragem Parcelas (DAP $\geq 10 \mathrm{~cm}$ ) & 120 & 250 & 30.000 & 1.417 & 261 & 51 \\
\hline Sub-amostragem $\mathrm{S} 1(5 \mathrm{~cm} \leq \mathrm{DAP} \leq 10 \mathrm{~cm})$ & 24 & 250 & 6.000 & 349 & 145 & 48 \\
\hline Sub-amostragem $\mathrm{S} 2(2 \mathrm{~cm} \leq \mathrm{DAP} \leq 5 \mathrm{~cm})$ & 24 & 100 & 2.400 & 460 & 161 & 48 \\
\hline Sub-amostragem S3 (DAP $>2 \mathrm{~cm}$ ) & 24 & 10 & 240 & 1.289 & 216 & 60 \\
\hline TOTAIS & & & & 3,515 & 471 & 77 \\
\hline
\end{tabular}

Obs: № de individuos por hectare: vide Tabela 11. 
Tabela 4. Similaridade e diversidade florística nas três unidades amostrais permanentes da floresta tropical primária, Município de Peixe-Boi, Pará.

\begin{tabular}{lccc}
\hline \multicolumn{1}{l}{ INDICE DE SIMILARIDADE (S) } & ÍNDICE DE DIVERSIDADE (H) \\
\hline SUA1, UA2 $=$ & 0,55 & HUA1 $=$ & 4,59 \\
SUA1, UA3 $=$ & 0,61 & HUA2 $=$ & 4,97 \\
SUA2, UA3 $=$ & 0,54 & HUA3 $=$ & 4,53 \\
\hline
\end{tabular}

florística entre as fitofisionomias estudadas calculou-se o indice qualitativo de Sörensen (1943) que representa a probabilidade de sortearse uma espécie comum às duas amostragens, considerando que elas têm o dobro de chance de serem amostradas do que as espécies que aparecem em apenas uma das amostragens.

Observa-se que a semelhança entre as unidades amostrais está por volta de $57 \%$; este valor, que a principio pode ser considerado alto quando comparado a outros estudos é, na verdade, relativamente baixo pois, sendo o fragmento de pequena área, esperava-se que a semelhança entre as UA's fosse maior. Tal fato pode ser decorrente da grande diversidade de espécies nas classes de DAP inferiores a $10 \mathrm{~cm}$, ou seja, no sub-bosque. Tal fato fica evidenciado quando se compara o índice de diversidade florística deste fragmento com o de outras florestas na Amazônia (Tab. 5).

Para a análise de diversidade foram utilizados os índices de Shannon \& Wiener (Ricklefs, 1979). O indice de Shannon \& Wiener $(\mathrm{H})$ é derivado da probabilidade de se obter uma seqüência de espécies pré-determinada contendo todas as espécies da amostra expresso pelo logarítmo neperiano da raiz enézima $\left(\mathrm{N}=\mathrm{n}^{\mathrm{e}}\right.$ total de indivíduos da amostra) desta probabilidade. Baseado na teoria da informação, seu valor mínimo ocorre quando todos os indivíduos pertencem à mesma espécie e o máximo quando cada indivíduo pertence a uma espécie diferente.

A eqüabilidade é a relação entre o índice de Shannon \& Wiener encontrado e o valor máximo possivel para o mesmo número de espécies, expresso quando todas as espécies apresentam o mesmo número de indivíduos, ou seja, é a propriedade de uma comunidade que diz respeito à uniformidade de distribuição de espécies ou suas abundâncias relativas. Equabilidade máxima significa uniformidade máxima e eqüabilidade mínima quando há uma espécie dominante. Por exemplo, uma amostra contendo 200 indivíduos em 10 espécies, com 20 individuos de cada espécie, tem mais eqüidade que uma amostra de mesmo tamanho e número de espécies, em que uma das espécies tem 100 indivíduos e as outras nove espécies compartilham os outros 100 indivíduos.

Um outro índice de diversidade (Simpson - D) expressa a probabilidade de quaisquer dois individuos, tomados aleatoriamente na 
Tabela 5. Valores de variảveis floristicas e estruturais de florestas primárias em estudos desenvolvidos em diversos locais da Amazônia brasileira.*

\begin{tabular}{|c|c|c|c|c|c|c|c|c|c|c|c|c|}
\hline FONTE & LOCAL & TIPO DE FLORESTA & $\begin{array}{l}\text { AMOSTRA } \\
\text { (ha) }\end{array}$ & $\begin{array}{l}\text { DAP } \\
(\mathrm{cm})\end{array}$ & $\begin{array}{c}\text { NO IND } \\
\text { ha: }\end{array}$ & $\begin{array}{c}\text { NO SPP } \\
\mathrm{ha}\end{array}$ & $\begin{array}{c}\text { NO FAM } \\
\text { ha }^{-1}\end{array}$ & $\begin{array}{c}\text { SPP } \\
\text { RARAS }[\%]\end{array}$ & H & $\begin{array}{c}A B \\
\left(m^{2} x^{\prime} h a^{\prime \prime}\right)\end{array}$ & $\begin{array}{c}\text { VOL } \\
\left(m^{3 x} \cdot h a\right)\end{array}$ & $\begin{array}{c}\text { BIOMASSA } \\
\left(1^{\circ} \mathrm{na} \mathrm{a}^{\prime}\right)\end{array}$ \\
\hline Bas tos (1948) & $S^{3} M^{2}=$ Vua Nova $(A P)$ & Terra Firme & 1,0 & 30 & & & & 47,9 & 3,58 & & & \\
\hline \multirow[t]{3}{*}{ Black et al (1950) } & Belém PA! & Igapó & 1,0 & 10 & & & & 36,7 & 2.63 & & & \\
\hline & Belém PA) & Terra Firme & 1,0 & 10 & 423 & 87 & $3 t$ & 37.9 & 3,72 & & & \\
\hline & Tefé (AM) & Terra Fime & 1,0 & 10 & & & & 53.2 & 3.86 & & & \\
\hline Pires ef al (1953) & Castanka(PA) & Terra Firme & 3.5 & 10 & 423 & $179^{\circ}$ & $47^{\circ}$ & 25,1 & 4,30 & & & \\
\hline Cain st $a t(1956)$ & Belëm (PA) & Terra Firme & 2,0 & 10 & 449 & $153^{\circ}$ & $39^{\circ}$ & 43,8 & 4.07 & 32,6 & & \\
\hline \multirow[t]{3}{*}{ Rodrigues (1963) } & Serra do Navio (AP) & Terra Firme & 2,6 & 15 & & & & 40,0 & 3.89 & & & \\
\hline & Serra do Navio (AP) & Terra Firme & 1,5 & 15 & $307^{*}$ & $78^{\circ}$ & & & & & 3243 & \\
\hline & Serra do Navio $(A P)$ & Terra Firne & 1.1 & 15 & $313^{\circ}$ & $80^{\circ}$ & & & & & 3728 & \\
\hline Prance et a' (1976) & Manaus (AM) & Terra Firme & 1,0 & is & 350 & 179 & 41 & 56,0 & 4,76 & & 2864 & \\
\hline Ponto et al (1976) & Manaus (AM) & Mata de-baxio & 1,0 & 10 & & & & 50,9 & 3,59 & & & \\
\hline Dantas et al (1980) & Capitāo $\left.P \propto C_{0} \circ P A\right]$ & Terra Fime & 1,0 & 9.55 & 504 & 121 & 39 & & & & 265,7 & \\
\hline Dantas \& Müler $(1980)$ & Transamazônica, km $23(\mathrm{PA})$ & Terra Fiłme & 1,0 & 9.55 & 577 & 101 & 29 & & & & 1863 & \\
\hline Dantas \& Müler (1980) & Transam az ónica, km $101(\mathrm{PA})$ & Terra Fime & 0.5 & 9,55 & $300^{\circ}$ & $899^{\prime}$ & $30^{\circ}$ & & & & $85.2^{*}$ & \\
\hline Lis boa \& Liscoa $\{1984\}$ & Rod. RO -429. km $90(R O)$ & Terra Fime & 1,0 & 9.55 & 593 & 128 & & & & 26.1 & 367.5 & \\
\hline Balée (1986) & Baxia fin Turiaçu (MA) & Terra Fime & 1,0 & 10 & 519 & 123 & $3 B$ & 48.8 & & & & \\
\hline \multirow[t]{2}{*}{ Camphell etal (1986) } & Bacia Rio Xingu (PA) & Terra Fime & 3,0 & 10 & 635 & $265^{\circ}$ & $39^{\circ}$ & 47.2 & 4,51 & $123.6^{n}$ & & \\
\hline & Bacia Rio Xingú (PA) & Vàrzea & 0,5 & 10 & & & & 45.0 & 2,81 & & & \\
\hline Siva et at $(1986)$ & Serra dos Carałàs (PA) & Terra Firme & 1.0 & 8.55 & 516 & 128 & 38 & 49.7 & 4,08 & 27,7 & 257,7 & \\
\hline Sabmâo el al (1988) & Sena dos Carajas $(P A)$ & Terra Fime & 1,0 & 10 & 484 & 122 & 39 & 36.1 & 4,23 & 21.6 & 2065 & 2460 \\
\hline alomầ \& Lisboa (1988) & JiParana $(R O)$ & Terra Firme & 1,0 & 9.55 & 573 & 171 & 43 & 49.7 & 4,44 & 31.1 & 271,1 & \\
\hline Salomăo (1991) & Matabe $\{P A\}$ & Teпra Fimme & 6,0 & to & 525 & $237^{\circ}$ & $46^{*}$ & 3.0 & & 27.9 & 2572 & 3162 \\
\hline Brown el al (1992) & Xapuri (AC) & Terra Fime & 0.5 & 20 & $126^{\circ}$ & & & & & 22,2 & & 320.0 \\
\hline Salomão \& Santos (1897) & Porto Tom belas $(P A)$ & Terra Firme solo argibso & 1.0 & 10 & 475 & 155 & 37 & 51,0 & & 32,8 & 2502 & 381,0 \\
\hline Sabmão $(2000)$ & Parto Tom belas $(P A)$ & Tetra Fime sob argibso & 1.0 & 10 & 474 & 186 & 43 & 58.0 & 4.71 & 30,5 & 365,6 & 3910 \\
\hline Salomá $(2000)$ & Porto Tombelas $\{P A\}$ & Tetra Firmesobo aren os o & 1,0 & 10 & 508 & 199 & 47 & 52.0 & 4,90 & 22.1 & 229.3 & 246.0 \\
\hline ESTE TRABALHO & PEXE BOI (PA) & TERRA FIRME & 3.0 & 10 & 467 & $261^{\circ}$ & $51^{\circ}$ & & 4,70 & 24.6 & 209.4 & 246,9 \\
\hline
\end{tabular}

*Valores relativos à área da amostra adotada pelo autor; DAP $(\mathrm{cm})=$ diămetro mínimo a 1,30 m do solo; $\mathrm{N}^{\circ}$ Ind ha" = número de indivíduos por hectare; $\mathrm{N}^{2}$ spp ha ${ }^{-1}=$ número de espécies por hectare; $\mathrm{N}^{\circ} \mathrm{Fam} \mathrm{ha}^{-1}=$ nümero de familias por hectare; $\mathrm{Spp}$ raras = espécies raras (espécie com um único individuo); $\mathrm{H}=$ índice de Shannon \& Wiener; $A B=$ área basal $\left(m^{2} \times h a^{-1}\right) ; \mathrm{Vol}=$ volume de madeira com casca $\left(\mathrm{m}^{3} \times \mathrm{xh}^{-1}\right)$; Biomassa: aèrea e viva (peso seco) para individuos com $\mathrm{DAP}{ }^{3} 10,0 \mathrm{~cm}$. 
amostra, pertencerem à mesma espécie (Simpson, 1949): $\mathrm{D}=1$ para uma monocultura e $\mathrm{D}=0$ para uma comunidade diversa composta de indivíduos únicos de cada espécie. Tal indice é muito influenciado pelas espécies mais abundantes e é insensivel àquelas espécies que aparecem com apenas um indivíduo na amostra (via de regra, na Amazônia oriental, temos observado que geralmente em uma amostra de 1,0 ha -DAP $\geq 10 \mathrm{~cm}$ - tem-se cerca de 450 indivíduos distribuidos em 130 a 140 espécies sendo que $1 / 3$ dessas espécies ocorrem com um único indivíduo 'espécies raras'). Este índice é pouco influenciado pela riqueza de espécies, pesando mais a abundância das espécies com maior freqüência. Magurran (1988) define o índice de Simpson como a probabilidade de dois individuos, tomados ao acaso, pertencerem à diferentes espécies: a expressão matemática apresentada a seguir é justamente a probabilidade de se tomar dois indivíduos da mesma espécie. O complementar (1 - D) é muitas vezes usado como índice de diversidade já que $\underline{\mathrm{D}}$ é um valor de dominância na comunidade - esta expressão é a probabilidade a que se refere Magurran (l.c.).

$\mathrm{O}$ indice de diversidade de espécies de Shannon \& Wiener, de acordo com Margalef (1972), normalmente apresenta valores entre 1,5 a 3,5 , raramente ultrapassando 4,5 para logaritmo neperiano; observa-se o intervalo calculado vai de 4,53 a 4,97. A eqüabilidade que também é alta indica que os valores de diversidade de Shannon \& Wiener são próximos ao máximo esperado para o número de espécies amostradas, o que demonstra que a grande maioria das espécies contribuem com números de indivíduos bem próximos nas UA's estudadas (Tab. 4).

Segundo Ducke \& Black (1954), é um fato estranhável que na hiléia (Amazônia) a longitude desempenhe um papel muito mais importante que a latitude na composição florística: a diferença das floras (expressa pelo $\mathrm{n}^{\mathrm{0}}$ de espécies) é muito mais acentuada entre Belém e Santarém que entre Belém e Caiena (Guiana Francesa) embora, no último caso, a distância seja maior. Todas as observações acusam número maior de espécies para o centro e noroeste da Amazônia que para as partes orientais e ocidentais da região (Tab. 5). A amostragem na floresta tropical de Peixe-Boi corrobora as observações de Ducke \& Black (1954). Contudo, deve-se atentar que o $\mathrm{n}^{\circ}$ de espécies não garante diferença na composição floristica; é necessário utilizar-se um índice de similaridade ou dissimilaridade: duas áreas com o mesmo $\mathrm{n}^{\circ}$ de espécies podem ser tão distintas quanto outras duas com diferentes $\mathrm{n}^{\text {os }}$ de espécies.

Praticamente metade das 77 famílias (37 ou 48,1\%), foram amostradas em todos os quatro estratos. Cerca de um terço (26 famílias ou $33,8 \%$ ) apresentaram espécies apenas em um estrato; outras oito (ou 10,4\%) famílias ocorreram em dois e as seis restantes $(7,8 \%)$ em três estratos (Tab. 6). 
Mimosaceae foi família de maior riqueza (44 espécies), seguindo-se Sapotaceae (41), Moraceae (40), Caesalpiniaceae (38) e Myrtaceae (36); 14 familias $(18,2 \%)$ ocorreram com uma única espécie sendo que metade delas apresentaram também um único indivíduo - estas famílias são as mais "frágeis" no avanço da fronteira agrícola, conseqüentemente, são as mais ameaçadas requerendo atenção redobrada num programa de conservação.

$\mathrm{Na}$ amostragem dos individuos com DAP $\geq 10 \mathrm{~cm}$ as famílias de maior riqueza foram Sapotaceae (19 espécies), Caesalpiniaceae (18), Mimosaceae (17) e Moraceae (16); com apenas uma espécie foram registradas oito (ou 15,7\%) familias (Tab. 6). Na sub-amostragem S1 as familias com maior diversidade foram Caesalpiniaceae (11 espécie) e Myrtaceae (10), na S2 foram Myrtaceae (11) e Mimosaceae e Burseraceae (10 cada) e, em S3 foram Bignoniaceae (20) e Mimosaceae (18). Apresentando uma única espécie foram observadas $22(45,8 \%), 19$ $(39,6 \%)$ e $14(23,3 \%)$ famílias em S1, S2 e S3 respectivamente.

As espécie arbóreas características do ecossistema florestal são mostradas na Tabela $7 \mathrm{com}$ alguns parâmetros fitossociológicos e os usos mais comuns da referida espécie.

Considerando-se as dez espécies de maior abundância nos estratos inferioires (Tab. 8), observa-se uma grande variação na composição floristica entre os estratos analisados. Todavia as duas espécies de maior abundância (Eschweilera coriacea e Lecythis idatimom), estão presentes, e com destaque, em quaisquer-dos estratos estudados (Cf. também Anexo 1 e Tab. 7).

Sinteticamente, com relação à composição florística do fragmento florestal primário, traduzida pelos resultados da amostragem das três parcelas permanentes ficou evidenciado que das 471 espécies identificadas 40 (ou $8,5 \%$ ) foram registradas nos quatro estratos (AP, $\mathrm{S} 1$, $\mathrm{S} 2$ e S3), enquanto a grande maioria das espécies (292 ou 62\%) foram exclusivas de um único estrato Anexo 1.

\section{Dinâmica da Florística}

Os valores do diferencial entre os anos 1 e 2, tanto da abundância de espécies quanto da riqueza das familias, independentemente da classe diamétrica, traduzem a dinâmica da vegetação florestal no período considerado (Tab. 9). Com relação aos totais apresentados nos respectivos anos de monitoramento houve aumento de $13 \%$ da abundância e de $3 \%$ da riqueza de espécies no ano 2 em relação ao ano 1 . Consequentemente, o recrutamento e o ingresso de espécies foram superiores $(66 \%$ e $21 \%$, respectivamente) à mortalidade e egresso de espécies no período considerado. Em outras palavras, 68 novas espécies ingressaram na amostra no Ano 2 em contraposição ao egresso (saída) de outras 56 também no Ano 2; das espécies registradas em ambos os anos 190 mantiveram a mesma 
Tabela 6. Riqueza (no de espécies) das familias registradas nas quatro amostragens das três UA's de floresta primária, Municipio de Peixe-Boi, Pará.

\section{FAMÍLIA}

1. Acanthaceae

2. Anacardiaceae

3. Annonaceae

4. Apocynaceae

5. Aquifoliaceae

6. Araceae

7. Araliaceae

8. Arecaceae

9. Bignoniaceae

10. Boraginaceae

11. Burseraceae

12. Caesalpiniaceae

13. Caricaceae

14. Caryocaraceae

15. Cecropiaceae

16. Celastraceae

17. Chrysobalanaceae

18. Clusiaceae

19. Combretaceae

20. Commeliaceae

21. Connaraceae

22. Convolvulaceae

23. Cucurbitaceae

24. Dichapetalaceae

25. Dilleniaceae

26. Ebenaceae

27. Elaeocarpaceae

28. Euphorbiaceae

29. Fabaceae

30. Flacourtiaceae

31. Gesneriaceae

32. Hernandiaceae

33. Hippocrateaceae

34. Humiriaceae

35. Icacinaceae

36. Indeterminada

37. Lauraceae

38. Lecythidaceae

39. Linaceae

\begin{tabular}{cccc}
\multicolumn{5}{c}{ № DE ESPÉCIES } \\
\hline AP & S1 & S2 & S3 \\
& & & 3 \\
4 & 1 & 1 & 2 \\
4 & 1 & 3 & 5 \\
10 & 7 & 7 & 9 \\
& 1 & & \\
& & & 7
\end{tabular}

1

$2 \quad 1 \quad 4$

$\begin{array}{llll}4 & 4 & 7 & 20\end{array}$

3

$\begin{array}{llll}12 & 6 & 10 & 5\end{array}$

$\begin{array}{llll}18 & 11 & 7 & 6\end{array}$

1

2

3

3

$\begin{array}{llll}10 & 6 & 2 & 6\end{array}$

$\begin{array}{llll}4 & 1 & 1 & 4\end{array}$

$\begin{array}{llll}5 & 3 & 1 & 1\end{array}$

2

1

1

NOTAS: $A P=$ amostragem das parcelas indivíduos com $\mathrm{DAP} \geq 10 \mathrm{~cm} ; \mathrm{S} 1=$ sub-amostragem individuos com 5 $\mathrm{cm} \leq \mathrm{DAP}<10 \mathrm{~cm} ; \mathrm{S} 2=$ sub-amostragem indivíduos $\mathrm{com} 2 \mathrm{~cm} \leq \mathrm{DAP}<5 \mathrm{~cm} ; \mathrm{S} 3=$ subamos-tragem individuos com DAP $<2 \mathrm{~cm}$.

\section{FAMÍLIA}

40. Loganiaceae

41. Malpighiaceae

42. Maranthaceae

43. Marcgraviaceae

44. Melastomataceae

45. Meliaceae

46. Menispermaceae

47. Mimosaceae

48. Moraceae

49. Musaceae

50. Myristicaceae

51. Myrsinaceae

52. Myrtaceae

53. Nyctaginaceae

54. Olacaceae

55. Orchidaceae

56. Piperaceae

57. Poaceae

58. Polygalaceae

59. Polygonaceae

60. Polypodiaceae

61. Quiinaceae

62. Rhizophoraceae

63. Rubiaceae

64. Rutaceae

65. Sapindaceae

66. Sapotaceae

67. Sellaginelaceae

68. Simarubaceae

69. Smilacaceae

70. Solanaceae

71. Sterculiaceae

72. Tiliaceae

73. Ulmaceae

74. Verbenaceae

75. Violaceae

76. Vochysiaceae

77. Zingiberaceae

TOTAL
NN DE ESPÉCIES

$\begin{array}{cccc}\text { AP } & \text { S1 } & \text { S2 } & \text { S3 } \\ 1 & 1 & 3 & 2 \\ 2 & 1 & 2 & 5 \\ & & & 8 \\ & 1 & & \\ 4 & 4 & 4 & 10 \\ 6 & 4 & 5 & 8 \\ & 1 & & 3 \\ 17 & 9 & 10 & 18 \\ 16 & 7 & 9 & 16\end{array}$

$\begin{array}{llll}2 & 2 & 2 & 6\end{array}$

$\begin{array}{llll}14 & 10 & 11 & 7\end{array}$

$\begin{array}{llll}2 & 1 & 3 & 3\end{array}$

$\begin{array}{llll}3 & 1 & 2\end{array}$

1

7

5

2

2

2

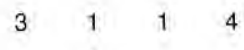

$\begin{array}{lll}2 & 3 & 11\end{array}$

2

$\begin{array}{llll}7 & 5 & 9 & 7\end{array}$

$\begin{array}{llll}19 & 7 & 9 & 9\end{array}$

$\begin{array}{lll}2 & 2 \\ & 2 & 1\end{array}$

1

$\begin{array}{llll}5 & 2 & 2 & 2\end{array}$

$\begin{array}{lll}3 & 1 & 1\end{array}$

$\begin{array}{llll}1 & 1 & 1 & 1\end{array}$

$\begin{array}{lll}1 & 1 & 1\end{array}$

$\begin{array}{llll}3 & 3 & 3 & 4\end{array}$

$\begin{array}{llll}4 & 1 & 3 & 3\end{array}$

2

60 
Tabela 7. Trinta espécies (DAP $\geq 10 \mathrm{~cm}$ ) com maiores valores do indice de importância ecológica (IVI) e respectivos uses, registradas nas três unidades amostrais permanentes do fragmento de floresta tropical primária, Município de Peixe-Boi, Pará.

\begin{tabular}{|c|c|c|c|c|c|c|}
\hline ESPÉCIE & NO IND & DR $(\%)$ & $\mathrm{FR}(\%)$ & DOR $(\%)$ & IVI (\%) & USOS \\
\hline 1. Eschweilera coriacea & 120 & 9,21 & 5,87 & 7,15 & 7,41 & $1-2-3-4$ \\
\hline 2. Lecythis idatimon & 96 & 7,13 & 5,32 & 5,36 & 5,94 & $1-3-4$ \\
\hline 3. Virola michelii & 27 & 1,49 & 1,53 & 4,67 & 2,56 & $1-2-3-4$ \\
\hline 4. Newtonia psilostachya & 13 & 1,11 & 1,35 & 5,1 & 2,52 & $1-6$ \\
\hline 5. Mabea aff. speciosa & 37 & 2,6 & 1,81 & 1,27 & 1,89 & $1-2-3$ \\
\hline 6. Apeiba burchellii & 25 & 2,08 & 1,81 & 1,43 & 1,77 & $1-2-4$ \\
\hline 7. Tapirira guianensis & 15 & 1,49 & 1,81 & 1,95 & 1.75 & 4 \\
\hline 8. Nectandra aff. globosa & 25 & 1,71 & 1,71 & 1,39 & 1,60 & $1-4-6$ \\
\hline 9. Poraqueiba guianensis & 28 & 1,78 & 1,81 & 0,66 & 1,42 & 4 \\
\hline 10. Goupia glabra & 8 & 0,52 & 0,63 & 3,02 & 1,39 & 1 \\
\hline 11. Vochysia vismiaefolia & 6 & 0,37 & 0,27 & 3,26 & 1,30 & $1-2$ \\
\hline 12. Sclerolobium paraense & 9 & 0,67 & 0,81 & 2,38 & 1,29 & 1 \\
\hline 13. Gustavia augusta & 29 & 1,86 & 1,35 & 0,38 & 1,20 & $1-3-4$ \\
\hline 14. Tachilia alba & 7 & 0,89 & 0,9 & 1,73 & 1,17 & 1 \\
\hline 15. Inga alba & 13 & 0,82 & 0,99 & 1,63 & 1,15 & $1-4-5-8$ \\
\hline 16. Jacaratia spinosa & 12 & 0,74 & 0,81 & 1,8 & 1,12 & $4-5-2$ \\
\hline 17. Protium sagotianum & 16 & 1,19 & 1,35 & 0,73 & 1,09 & $2-4-7$ \\
\hline 18. Lecythis pisonis & 8 & 0,67 & 0,63 & 1,92 & 1,07 & $1-3-4-5$ \\
\hline 19. Geissospermum sericeum & 7 & 1,04 & 1,26 & 0,85 & 1,05 & 2 \\
\hline 20. Pogonophora schomburgkiana & 10 & 0,82 & 0,9 & 1,43 & 1,05 & $1-4$ \\
\hline 21. Apuleia leiocarpa & 9 & 0,74 & 0,72 & 1,52 & 0,99 & 1 \\
\hline 22. Erisma unicinatum & 8 & 0,52 & 0,63 & 1,73 & 0,96 & $1-4$ \\
\hline 23. Newtonia suaveolens & 11 & 0,82 & 0,81 & 1,23 & 0,95 & $1-6$ \\
\hline 24. Symphonia globulifera & 19 & 1,04 & 1,17 & 0,65 & 0,95 & $1-2-9$ \\
\hline 25. Micropholis guianensis & 8 & 0,97 & 0,99 & 0,87 & 0,94 & $1-4-9$ \\
\hline 26. Eschweilera aff. collina & 9 & 0,74 & 0,81 & 1,18 & 0,91 & $1-2-4$ \\
\hline 27. Mouriri brachyanthera & 14 & 1,11 & 1,17 & 0,44 & 0,91 & $1-2$ \\
\hline 28. Cecropia distachya & 14 & 1,49 & 0,54 & 0,64 & 0,89 & 4 \\
\hline 29. Trichilia micrantha & 12 & 1,04 & 1,17 & 0,41 & 0,87 & 4 \\
\hline 30. Protium tenuifolium & 11 & 0,82 & 0,9 & 0,84 & 0,85 & $1-4-7$ \\
\hline TOTAL & 626 & 41,83 & 47,48 & 57,62 & 48,98 & \\
\hline
\end{tabular}

NOTAS: No ind=número de individuos; $\mathrm{DR}=$ densidade relativa; $\mathrm{FR}=$ frequência relativa; $\mathrm{DoR}=$ dominância relativa, IVI= índice de valor de importância. USOS: 1 -Madeira comercial; 2-Medicinal; 3-Fibras; 4- Alimento para fauna; 5- Alimento para o homem; 6- Óleo essencial; 7-Resina; 8-Tintura e, 9- Látex. 
Tabela 8. Estimativas do número de indivíduos por hectare das dez espécies mais abundantes nas três sub-amostragens da floresta tropical primária densa, Município de Peixe-Boi, Pará.

\begin{tabular}{|c|c|c|c|c|c|c|c|c|}
\hline \multicolumn{3}{|c|}{ SUB-AMOSTRAGEM $S 1(5 \mathrm{~cm}>$ DAP $>10 \mathrm{~cm})$} & \multicolumn{3}{|c|}{$\begin{array}{c}\text { SUA-AMOSTRAGEM S2 }(2 \mathrm{~cm} \geq \text { DAP }>5 \mathrm{~cm}) \\
-\end{array}$} & \multicolumn{3}{|c|}{ SUE.AMOSTRAGEM S3 $\{$ DAP $<2 \mathrm{~cm}\rangle$} \\
\hline \multirow[t]{2}{*}{ ESPECEE } & \multicolumn{2}{|c|}{ NO $\mathbb{N O}_{\mathrm{A}}$ ha } & \multirow[t]{2}{*}{ ESPÉCIE } & \multicolumn{2}{|c|}{ NO IND • ha 1} & \multirow[t]{2}{*}{ ESPÉCIE } & \multicolumn{2}{|c|}{ NO IND ' b ' } \\
\hline & ANO: & ANO2 & & ANO 1 & ANO2 & & ANO 1 & ANO2 \\
\hline Gustavia augusta & 190 & 190 & Rinorea neglecta & 575 & 613 & Eschweilera coriacea & 44.000 & 5.875 \\
\hline Sterculia pruriens & 55 & 50 & Amphirrox ct. surnamensis & 313 & 325 & Lecylhis idatimon & 6.375 & 16.875 \\
\hline Eschueitera coriacea & 40 & 50 & Memora flavida & 300 & 288 & Mabea aft. speciosa & 5.750 & 26.750 \\
\hline Geissospermum sericeum & 40 & 45 & Protium pitosum & 225 & 188 & Poraqueiba guianensis & 3.875 & 1.500 \\
\hline Eauhinia guianensis & 40 & 45 & Neoptychocarpus apodansthus & 200 & 250 & Gustavia augusta & 3.250 & 2.875 \\
\hline Protiem trifotiofatum & 40 & 40 & Bauthinia ct. rutitans & 150 & 100 & Nectandra aff, globosa & 3.000 & 3.625 \\
\hline Hippocratea ct. ovata & 40 & 40 & Eschweilera coriacea & 138 & 125 & Virola michelii & 2.125 & 2.750 \\
\hline Calyptranthes cf. macrophylta & 35 & 40 & Gustavia altgusta & 125 & 150 & Sympironla giobutifera & 1.875 & 12.250 \\
\hline Lecythis idatimon & 35 & 35 & Memora afamanditfora & 125 & 125 & Licania carnescens & 1.750 & 2.875 \\
\hline Virola michelif & 35 & 30 & Neea spi & 88 & 100 & Mouniri brachyanthera & 1.625 & 3.000 \\
\hline TOTAIS & 550 & 565 & TOTAIS & 2.238 & 2.263 & TOTALS & 73.625 & 78.375 \\
\hline
\end{tabular}

abundância enquanto 103 aumentaram-na, face a outras 54 espécies que apresentaram declínio do número de indivíduos (Anexo 1).

A variação da riqueza arbórea (DAP $\geq 10 \mathrm{~cm}$ ) mostrou-se, no intervalo de um ano, sensivelmente dinâmica pois houve um incremento de $3,2 \%$ no número de espécies, ou seja, um acréscimo de oito novas espécies; não foi registrado nenhum egresso entre as 253 espécies inicialmente registradas. Em sintese, houve um ganho líquido de oito novas espécies nas três UA's (Tab. 9).

Por outro lado, no sub-bosque, nas subamostragens $\mathrm{S} 1$ e S2 $(10 \mathrm{~cm}>\mathrm{DAP} \geq$ $2 \mathrm{~cm}$ ) o ganho líquido foi nulo. Todavia a dinâmica da composição florística ficou altamente evidenciada: 11 e 14 espécies saíram da amostra respectivamente, naquelas subamostragens, contra igual número de ingressos para o mesmo periodo. Já com relação ao número de famílias houve um ganho líquido de duas famílias em S1 e de uma familia em S2
(Tab. 9). Estes dados demonstram que a dinâmica é diferenciada entre os diversos estratos da floresta; conseqüentemente, conhecer a composição do sub-bosque não permite projetar a composição florística futura do dossel.

Considerando-se a amostragem global (todos as classes diamétricas nos três ha de parcelas permanentes) verificou-se, no prazo de um ano, o egresso de 56 espécies versus o ingresso de 68 espécies, gerando um ganho liquido de 12 espécies; já o número de famílias permaneceu constante durante o periodo analisado.

A dinâmica da regeneração natural stricto sensu (sub-amostragem S3) demonstra notável variação florística, tanto a nível de espécies como de familias - resultado esperado devido a maior mortalidade nesta fase e a germinação e estabelecimento de novas plântulas. No período de apenas um ano houve um incremento positivo de mais de um quarto do número total de espécies ou, em outras palavras, um 
Tabela 9. Dinâmica da abundância $\mathrm{e}$ da riqueza das espécies $\mathrm{e}$ familias, por classe diamétrica, nas três parcelas permanentes do fragmento de floresta tropical primária; Município de Peixe-Boi, Pará.

NÚMERO DE INDNIDUOS

CLAS SE DE DIÂMETRO
NUMERO DE ESPÉCIES

NUMERO DE FAMILIS
ABUNDÂNCIA

MORTALIDADE' RECRUTAMENTO
- IJ-LI
ANO1 ANO2

ANO1

ANO2
EGRESSOS INGRESSOS

ANO1 ANO2

$D A P \geq 10.0 \mathrm{~cm}^{2}$

$(2,0 \%)$

60

$(4,3 \%)$

253

261

(0\%)

8

$(3,2 \%)$

51

$\%)$

$10.0 \mathrm{~cm}>D A P \geq 5,0 \mathrm{~cm}^{(3)}$

$5.0 \mathrm{~cm}>$ DAP $\geq 2,0 \mathrm{~cm}$ N

$$
458
$$

$$
(10.5 \%)
$$

DAP $<2.0 \mathrm{~cm}$

$1.289 \quad 503$

$(54,2 \%)$

$-1$.

NOTAS: ${ }^{(t)}$ do período (corrente);valores percentuais calculados em relaçāo ao Ano 1

(2) valores referentes a $3,0 \mathrm{ha}$.

13) valores referentes a $0,6 \mathrm{ha}$.

(4) valores referentes para $0,24 \mathrm{ha}$.

15) valores referentes para 0,024 ha 
acréscimo de $57 \%$ de 'novas' espécies sobre o número de espécies que saíram da amostra (Tab. 9). Em relação ao número famílias, esses valores foram ainda mais supreendentes: contra uma família que saiu outras 14 ingressaram, ou seja, houve um enriquecimento de mais de $28 \%$ no número de famílias. Percebe-se um surpreendente ganho líquido de 13 famílias e 33 espécies no período de apenas um ano (Fig. 1). Todavia, tal fato não permite maiores conclusões sobre a expansão do fragmento, pois como demonstrado, a dinâmica neste nível é intensa e pode ser inversa no próximo ano; necessita-se de um período longo de observações para se concluir algo à respeito.

A dinâmica da riqueza e da abundância intrínseca a cada família, independentemente da classe diamétrica dos indivíduos registrados (Tab. 10 e Anexo 1), permite inferir que no período de uma ano:

Trinta e três famílias (ou 43\%) apresentaram crescimento do número de espécies, em oposição a 15 (ou 19\%) que apresentaram declínio; 29 (ou 38\%) mantiveramse constantes.

Malpighiaceae (três espécies), Meliaceae, Myrtaceae e Sapindaceae ( 2 espécies cada) e outras 10 familias (uma espécie cada) apresentaram egresso de espécies; outras 28 familias mantiveram inalterada a diversidade de espécies.

O maior ingresso de espécies foi observado em Lauraceae (5 espécies), Arecaceae e Sapotaceae (4 cada), outras 4, 11 e 17 famílias apresentaram, respectivamente, $3,2 \mathrm{e}$
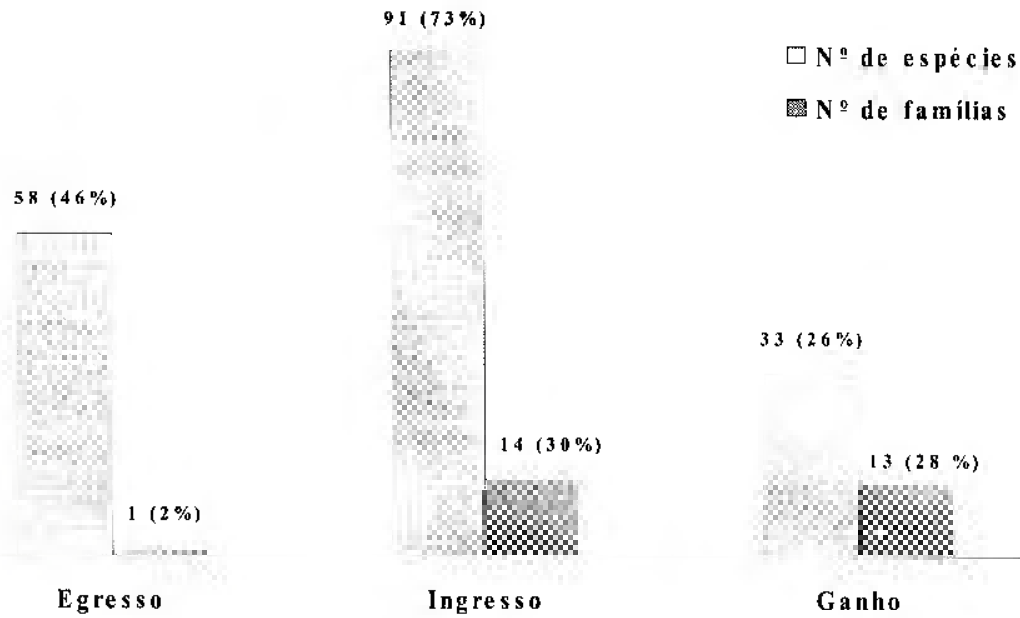

$33\{26 \%\}$ $13(28 \%)$

Figura 1. Dinâmica das famílias e espécies registradas na regeneração natural (S3), no periodo de 12 meses nas três parcelas permanentes do fragmento de floresta tropical primária, Município de Peixe-Boi, Pará. Os números entre parêntesis referem-se ao percentual em relação ao total do referido periodo. 
1 espécies ingressadas.

Relativamente à abundância, 48 famílias (ou 62\%) tiveram acréscimo do número de indivíduos, outros $15 \%$ apresentaram declínio, enquanto $18 \%$ (ou 14) mantiveramse constantes.

Caesalpiniaceae foi a familia que apresentou a maior mortalidade absoluta (228 individuos); outras 14 familias perderam entre 1 a 24 indivíduos.

Violaceae (181 indivíduos) e Lecythidaceae (106) apresentaram o maior recrutamento absoluto; outras 46 famílias recrutaram entre 1 e 83 indivíduos.

Catorze familias não apresentaram variação na abundância de individuos.

A dinâmica das espécies, nas diferentes classes diamétricas amostradas, no período de um ano, permite observar que (Anexo 1):

Na classe de DAP $\geq 10 \mathrm{~cm}$ : (a) 21 espécies apresentaram mortalidade de um individuo enquanto Cecropia distachya apresentou uma mortalidade de cinco individuos e Mabea aff. speciosa de dois - sendo espécies pioneiras apresentam maior mortalidade quando do fechamento do dossel; (b) 199 espécies não apresentaram alteração do número de individuos; (c) 28 espécies apresentaram recrutamento de um indivíduo, enquanto outras oito apresentaram de dois; (d) Bauhinia cf rutilans (sete indivíduos), Lecythis idatimom (seis) e Protium trifoliolatum (três) foram as espécies que apresentaram maior recrutamento.
$\mathrm{Na}$ classe de $10 \mathrm{~cm}>$ DAP $\geq$ $5 \mathrm{~cm}$ : (a) 22 espécies apresentaram mortalidade de um indivíduo; (b) 94 espécies mantiveram a mesma abundância no período considerado; (c) 24 espécies apresentaram recrutamento de um indivíduo; (d) 33 espécies apresentaram indivíduos em ambos os anos considerados, $\mathrm{cm}$ contraposição a, respectivamente, 41 e 71 espécies que apresentaram indivíduos apenas nos anos 1 e 2 .

$\mathrm{Na}$ classe de $5 \mathrm{~cm}>$ DAP $\geq 2$ $\mathrm{cm}$ : (a) 35 espécies apresentaram mortalidade de um indivíduo enquanto Bauhinia cf rutilans e Protium pilosum apresentaram maior mortalidade - quatro e três indivíduos, respectivamente; (b) 82 espécies mantiveram a mesma abundância; (c) 33 espécies recrutaram um indivíduo, já o maior recrutamento foi de Neoptychocarpus apodanthus - 4 indivíduos; (d) 42 espécies apresentaram individuos em ambos períodos analisados, em contraste com 61 e 58 espécies que apresentaram indivíduos apenas no Ano 1 e no Ano 2 , respectivamente.

$\mathrm{Na}$ classe de DAP $<2 \mathrm{~cm}$ : (a) 36 espécies apresentaram mortalidade de um indivíduo enquanto em outras 35 variou de 2 a 12 indivíduos; (b) Bauhinia cf rutilans e Mabea aff speciosa foram as espécies de maior mortalidade 305 e 19 indivíduos, respectivamente; (c) apenas 12 espécies mantiveram abundância constante; (d) 44 espécies recrutaram um indivíduo face a outras 83 espécies que oscilaram entre 2 a 22 individuos; 
(e) Rinorea neglecta, Calathea sp, Lecythis idatimom e Bauhinia cuprionites com respectivamente, 168 , 84,83 e 77 indivíduos foram as espécies que apresentaram maiores recrutamentos.

Finalmente, considerando a amostragem total, independentemente da classe diamétrica, temse: (a) Bauhinia cf rutilans e Mabea aff speciosa apresentaram maior mortalidade absoluta: 300 e 21 individuos, respectivamente; (b) o maior recrutamento foi obtido por Rinorea neglecta e Lecythis idatimom: 171 e 89 espécimes respectivamente.

\section{Dinâmica da Estrutura}

Nas parcelas e subparcelas S1, S2 e S3 foi calculada uma mortalidade de respectivamente, $2,0 \%, 7,5 \%, 10,5 \%$ e $54,2 \%$; por outro lado o recrutamento no mesmo periodo foi de $4,3 \%, 8,7 \%$, $10,9 \%$ e $93,1 \%$ (Tab. 9), ou seja, o quociente recrutamente/mortalidade foi, em quaisquer das classes diamétricas sempre superior a unidade, o que demonstra uma tendência de crescimento dos individuos como um todo com conseqüente aporte de biomassa ao ecossistema, caso tal fato se confirme a longo prazo.

Os altos valores da abundância e diversidade observados na subamostragem $\mathrm{S} 3$ era esperado pois trata-se do estrato inferior da floresta, onde a regeneração natural e a mortalidade são muito intensas, acarrretando uma densidade muita elevada de individuos por unidade de área, neste caso, média de 138.625 individuos por ha, correspondendo a mais de $97 \%$ do total de individuos - Figura 2.

A seleção natural ocorre de forma continua no ecossistema atuando durante todos os processos da regeneração natural. Observou-se neste estudo que para cada árvore com limite mínimo de $10 \mathrm{~cm}$ de DAP necessitou-se de um estoque de 297 mudas por ha, para cada arvoreta (DAP entre 5 e $10 \mathrm{~cm}$ ) houve uma necessidade de 160 mudas por ha $\mathrm{e}$ que para cada vara (DAP entre 2 e 5 $\mathrm{cm}$ ) o estoque seria de 48 mudas por ha. Obviamente, estas estimativas são uma aproximação em referência ao estoque bruto, onde se pressupõe que todas as mudas amostradas em S3 teriam a mesma chance de chegar ao estágio de árvores (DAP $\geq 10 \mathrm{~cm}$ ) o que sabe-se, não é verdade, pois muitas espécies, na fase adulta, têm porte arbustivo ou herbáceo, ou seja, não ultrapassam o limite de $10 \mathrm{~cm}$ de DAP para que sejam enquadradas na categoria de árvores.

A área basal dos individuos com DAP $\geq 10 \mathrm{~cm}$ apresentou um incremento positivo de $0,9 \mathrm{~m}^{2} * \mathrm{ha}^{-1}$ assim como os individuos das sub-amostragem S1, só que não nesta magnitude; na subamostragem S2 não houve alteração no perído considerado (Tab. 11). A comparação destes valores com os de outras florestas na Amazônia brasileira é apresentada na Tabela 5.

\section{Biomassa}

A produtividade primária dos ecossistemas florestais tropicais é maior do que a de climas temperados, 
Tabela 10. Dinâmica da diversidade e da abundância arbórea, por familia, em três ha de parcelas permanentes em floresta tropical primária, Municipio de Peixe-Boi, Pará.

\begin{tabular}{|c|c|c|c|c|c|c|}
\hline \multirow{2}{*}{$\begin{array}{c}\text { FAMILIA } \\
\therefore\end{array}$} & \multicolumn{3}{|c|}{$\begin{array}{l}\text { RIQUEZA ARBÓREAN de } \\
\text { Espécies * ha*1 }\end{array}$} & \multicolumn{3}{|c|}{$\begin{array}{l}\text { ABUNDÂNCIA ARBÓREAN" } \\
\text { de Indivíduos } \text { " ha }^{*}\end{array}$} \\
\hline & 1998 & 1999 & $\Delta 98,99$ & 1998 & 1999 & $\Delta 98,99$ \\
\hline 1. Acanthaceae & 1 & 2 & $\uparrow$ & 5 & 4 & $\downarrow$ \\
\hline 2. Anacardiaceae & 6 & 8 & $\uparrow$ & 36 & 37 & $\uparrow$ \\
\hline 3. Annonaceae & 7 & 10 & $\uparrow$ & 16 & 29 & $\uparrow$ \\
\hline 4. Apocynaceae & 26 & 28 & $\uparrow$ & 74 & 81 & $\uparrow$ \\
\hline 5. Aquifoliaceae & 1 & 1 & K & 1 & 1 & $\mathbf{K}$ \\
\hline 6. Araceae & 4 & 3 & $\downarrow$ & 16 & 7 & $\downarrow$ \\
\hline 7. Araliaceae & 1 & 1 & $\mathbf{K}$ & 6 & 6 & $\mathbf{K}$ \\
\hline 8. Arecaceae & 3 & 7 & $\uparrow$ & 6 & 10 & $\uparrow$ \\
\hline 9. Bignoniaceae & 22 & 24 & $\uparrow$ & 86 & 112 & $\uparrow$ \\
\hline 10. Boraginaceae & 3 & 3 & K & 9 & 10 & $\uparrow$ \\
\hline 11. Burseraceae & 30 & 31 & $\uparrow$ & 138 & 167 & $\uparrow$ \\
\hline 12. Caesalpiniaceae & 37 & 38 & $\uparrow$ & 494 & 266 & $\downarrow$ \\
\hline 13. Caricaceae & 1 & 1 & $\mathrm{~K}$ & 12 & 12 & K \\
\hline 14. Caryocaraceae & 3 & 3 & $\mathrm{~K}$ & 6 & 6 & $\mathbf{K}$ \\
\hline 15. Cecropiaceae & 4 & 4 & K & 23 & 18 & $\downarrow$ \\
\hline 16. Celastraceae & 4 & 3 & $\downarrow$ & 21 & 20 & $\downarrow$ \\
\hline 17. Chrysobalanaceae & 21 & 20 & $\downarrow$ & 67 & 64 & $\downarrow$ \\
\hline 18. Clusiaceae & 7 & 9 & $\uparrow$ & 36 & 45 & $\uparrow$ \\
\hline 19. Combretaceae & 9 & 8 & $\downarrow$ & 21 & 19 & $\downarrow$ \\
\hline 20. Commeliaceae & 1 & 1 & $\mathrm{~K}$ & 1 & 7 & $\uparrow$ \\
\hline 21. Connaraceae & & 1 & $\uparrow$ & & 1 & $\uparrow$ \\
\hline 22. Convolvulaceae & 1 & 1 & $\mathrm{~K}$ & 1 & 1 & $\mathrm{~K}$ \\
\hline 23. Cucurbitaceae & & 2 & $\uparrow$ & & 2 & $\uparrow$ \\
\hline 24. Dichapetalaceae & & 3 & $\uparrow$ & & 4 & $\uparrow$ \\
\hline 25. Dilleniaceae & 9 & 11 & $\uparrow$ & 19 & 26 & $\uparrow$ \\
\hline 26. Ebenaceae & 4 & 5 & $\uparrow$ & 10 & 13 & $\uparrow$ \\
\hline 27. Elaeocarpaceae & 5 & 6 & $\uparrow$ & 11 & 13 & $\uparrow$ \\
\hline 28. Euphorbiaceae & 18 & 17 & $\downarrow$ & 112 & 88 & $\downarrow$ \\
\hline 29. Fabaceae & 18 & 17 & $\downarrow$ & 22 & 21 & $\downarrow$ \\
\hline 30. Flacourtiaceae & 7 & 7 & K & 48 & 59 & $\uparrow$ \\
\hline 31. Gesneriaceae & & 1 & $\uparrow$ & & 9 & $\uparrow$ \\
\hline 32. Hernandiaceae & 2 & 3 & $\uparrow$ & 2 & 3 & $\uparrow$ \\
\hline 33. Hippocrateaceae & 12 & 14 & $\uparrow$ & 34 & 50 & $\uparrow$ \\
\hline 34. Humiriaceae & 4 & 3 & $\downarrow$ & 6 & 4 & $\downarrow$ \\
\hline 35. Icacinaceae & 7 & 7 & $\mathrm{~K}$ & 49 & 46 & $\downarrow$ \\
\hline 36. Indeterminada & 2 & 2 & $\mathbf{K}$ & 2 & 2 & $\mathbf{K}$ \\
\hline 37. Lauraceae & 14 & 19 & $\uparrow$ & 65 & 98 & $\uparrow$ \\
\hline 38. Lecythidaceae & 25 & 27 & $\uparrow$ & 420 & 526 & $\uparrow$ \\
\hline
\end{tabular}


Tabela 10. Continuação

\begin{tabular}{|c|c|c|c|c|c|c|}
\hline \multirow[t]{2}{*}{ IAH _L } & \multicolumn{3}{|c|}{$\begin{array}{l}\text { RIQUEZA ARBÓREAN№ de } \\
\text { Espécies * }{ }^{*} \mathrm{ha}^{-1}\end{array}$} & \multicolumn{3}{|c|}{$\begin{array}{l}\text { ABUNDÂNCIA ARBÓREANe de } \\
\text { indivíduos * ha:l }\end{array}$} \\
\hline & 1998 & 1999 & $\Delta 98,99$ & 1998 & 1999 & $\Delta 98,99$ \\
\hline 39. Linaceae & 1 & 1 & $\mathrm{~K}$ & 1 & 1 & K \\
\hline 40. Loganiaceae & 5 & 6 & $\uparrow$ & 8 & 8 & K \\
\hline 41. Malpighiaceae & 9 & 6 & $\downarrow$ & 24 & 15 & $\downarrow$ \\
\hline 42. Maranthaceae & 3 & 5 & $\uparrow$ & 65 & 148 & $\uparrow$ \\
\hline 43. Marcgraviaceae & 1 & 1 & K & 1 & 1 & $\mathrm{~K}$ \\
\hline 44. Melastomataceae & 15 & 17 & $\uparrow$ & 63 & 71 & $\uparrow$ \\
\hline 45. Meliaceae & 20 & 18 & $\downarrow$ & 75 & 76 & $\uparrow$ \\
\hline 46. Menispermaceae & 2 & 2 & $\mathrm{~K}$ & 2 & 3 & $\uparrow$ \\
\hline 47. Mimosaceae & 41 & 44 & $\uparrow$ & 145 & 172 & $\uparrow$ \\
\hline 48. Moraceae & 37 & 40 & $\uparrow$ & 80 & 85 & $\uparrow$ \\
\hline 49. Musaceae & & 1 & $\uparrow$ & $i$ & 4 & $\uparrow$ \\
\hline 50. Myristicaceae & 9 & 9 & $\mathrm{~K}$ & 59 & 61 & $\uparrow$ \\
\hline 51. Myrsinaceae & 1. & & $\downarrow$ & 7 & & $\downarrow$ \\
\hline 52. Myrtaceae & 38 & 36 & $\downarrow$ & 82 & 77 & $\downarrow$ \\
\hline 53. Nyctaginaceae & 7 & 8 & $\uparrow$ & 30 & 39 & $\uparrow$ \\
\hline 54. Oiacaceae & 9 & 9 & $\mathrm{~K}$ & 27 & 27 & K \\
\hline 55. Orchidaceae & & 1 & $\uparrow$ & & 1 & $\uparrow$ \\
\hline 56. Piperaceae & 4 & 3 & $\downarrow$ & 7 & 15 & $\uparrow$ \\
\hline 57. Poaceae & 3 & 2 & $\downarrow$ & 15 & 28 & $\uparrow$ \\
\hline 58. Polygalaceae & 1 & 1 & $\mathrm{~K}$ & 1 & 6 & $\uparrow$ \\
\hline 59. Polygonaceae & 3 & 3 & $\mathrm{~K}$ & 7 & 14 & $\uparrow$ \\
\hline 60. Polypodiaceae & 1 & 1 & K & 1 & 1 & $\mathrm{~K}$ \\
\hline 61. Quiinaceae & 7 & 7 & $\mathrm{~K}$ & 24 & 25 & $\uparrow$ \\
\hline 62. Rhizophoraceae & 1 & 1 & $\mathrm{~K}$ & 1 & 2 & $\uparrow$ \\
\hline 63. Rubiaceae & 13 & 14 & $\uparrow$ & 31 & 48 & $\uparrow$ \\
\hline 64. Rutaceae & 2 & 2 & $\mathrm{~K}$ & 6 & 6 & $\mathrm{~K}$ \\
\hline 65. Sapindaceae & 25 & 23 & $\downarrow$ & 70 & 78 & $\uparrow$ \\
\hline 66. Sapotaceae & 37 & 41 & $\uparrow$ & 111 & 124 & $\uparrow$ \\
\hline 67. Sellaginelaceae & 1 & 1 & $\mathrm{~K}$ & 5 & 3 & $\downarrow$ \\
\hline 68. Simarubaceae & 4 & 5 & $\uparrow$ & 11 & 13 & $\uparrow$ \\
\hline 69. Smilacaceaeae & & 1 & $\uparrow$ & & 3 & $\uparrow$ \\
\hline 70. Solanaceae & 1 & 1 & K & 2 & 2 & $\mathrm{~K}$ \\
\hline 71. Sterculiaceae & 10 & 10 & $\mathrm{~K}$ & 45 & 48 & $\uparrow$ \\
\hline 72. Tiliaceae & 5 & 5 & $\mathrm{~K}$ & 36 & 36 & $\mathrm{~K}$ \\
\hline 73. Ulmaceae & 3 & 4 & $\uparrow$ & 15 & 22 & $\uparrow$ \\
\hline 74. Verbenaceae & 2 & 3 & $\uparrow$ & 2 & 3 & $\uparrow$ \\
\hline 75. Violaceae & 10 & 12 & $\uparrow$ & 130 & 311 & $\uparrow$ \\
\hline 76. Vochysiaceae & 9 & 9 & $\mathrm{~K}$ & 43 & 48 & $\uparrow$ \\
\hline 77. Zingiberaceae & 1 & 1 & $\mathrm{~K}$ & 3 & 13 & $\uparrow$ \\
\hline TOTAIS & 70 & 76 & - & 3.116 & 3.515 & - \\
\hline
\end{tabular}

Convenção: D 98,92 [variação do ano inicial (t998) ao ano final (1999)]; $\uparrow=\overline{\text { crescente; } \downarrow=\text { decrescente; } K}$ $=$ constante. 


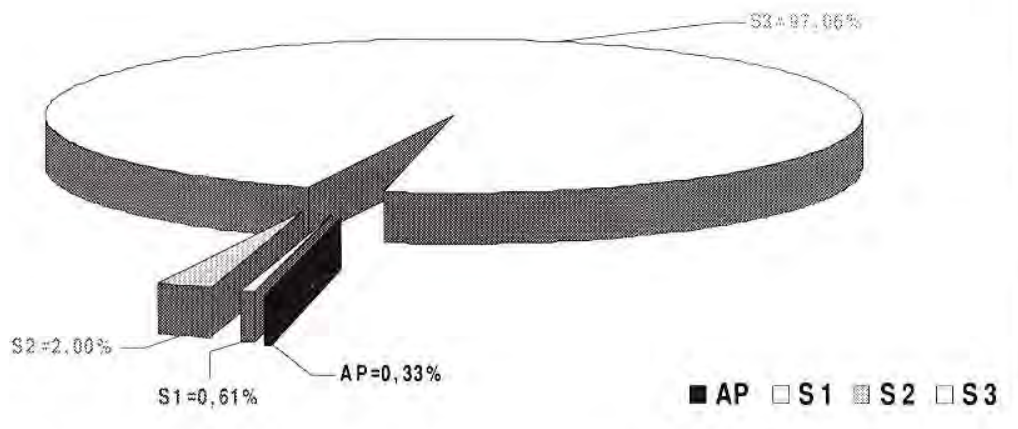

Figura 2 - Percentual médio do número de indivíduos por hectare de acordo com a classe diamétrica: AP (DAP $\geq 10 \mathrm{~cm}) ; \mathrm{S} 1(10 \mathrm{~cm}>\mathrm{DAP} \geq 5 \mathrm{~cm}) ; \mathrm{S} 2(5 \mathrm{~cm}>$ DAP $\geq 2 \mathrm{~cm})$ e S3 (DAP $<2 \mathrm{~cm})$. Obs: números absolutos, vide Tabela 11.

embora os solos tropicais sejam menos férteis. Nódulos radiculares (retenção e transferência de p. ex: nitrogênio), micorrizas, retenção e velocidade de ciclagem de nutrientes na biomassa e imobilização de nutrientes na serrapilheira estariam contribuindo decisivamente na produção primária.

A eliminação de florestas tropicais primárias contribui significativamente para o aumento de dióxido de carbonbo $\left(\mathrm{CO}_{2}\right)$ na atmosfera, acarretando importantes variações climáticas. Qual o estoque de carbono da biomassa das diferentes tipologias florestais amazônicas? Das florestas que recobriam majoritariamente a região Bragantina têm-se hoje menos de $2 \%$ de remanescentes florestais.

A biomassa aérea viva das árvores (DAP $\geq 10 \mathrm{~cm})$ apresentou incremento corrente anual (ICA) de $4,3 \mathrm{t}^{*} \mathrm{ha}^{-1 *}$ ano $^{-1}$; em S1 o ICA foi de $0,1 \mathrm{t}^{*} \mathrm{ha}^{-1}$ ano $^{-1}$ (Tab. 11). A biomassa estimada no fragmento de floresta primária de Peixe-Boi encontra-se próxima da média para a Amazônia oriental (Tab. 5).

\section{Conclusão}

Este estudo permite inferir que em um hectare de floresta primária densa da Amazônia oriental tem-se c.a. 143.000 indivíduos, desde plântulas até árvores e, no mínimo, algo como 337 espécies e 76 famílias de Dicotiledôneas.

A riqueza florística (números de espécies e familias) da floresta do nordeste do Pará, especificamente de Peixe-Boi, tem valores próximos a média da Amazônia oriental que é inferior à parte ocidental.

Mimosaceae foi família de maior riqueza, com 44 espécies, seguindo-se Sapotaceae (41), Moraceae (40), Caesalpiniaceae (38) e Myrtaceae (36); 14 famílias $(18,2 \%)$ ocorreram 
Tabela 11. Dinâmica e respectivos valores médios, por hectare, da abundância (no de indivíduos $\left.{ }^{*} \mathrm{ha}^{-1}\right)$, dominância $\left(\mathrm{m}^{2 *} \mathrm{ha}^{-1}\right)$, biomassa aérea viva e volume de madeira nas respectivas diversas classes diamétricas da vegetação do fragmento de floresta tropical primária, Municipio de Peixe-Boi,

Dará

CLASSE DIA METRICA (DAP)

$\frac{\text { NUMERO DE INDIVIDUOS*HA " }}{\text { ANO } 1 \text { ANO } 2 \quad \text { ICA }}$

AREA BASAL $\left(M^{2+} \mathrm{HA}^{1}\right)$

BIOMASSA (T*HA $\left.{ }^{1}\right)$

VOLUME $\left(\mathrm{M}^{3 \cdot} \mathrm{HA}^{-1}\right\}$

ANO 1 ANO $2 \quad$ ICA

ANO 1 ANO 2

ICA

ANO 1 ANO2

Amostragem das Parcelas (DAP $\geq 10 \mathrm{~cm}$ )

Sub-am ostragem $S 1(5 \mathrm{~cm} \leq D A P \leq 10 \mathrm{~cm})$

Sub-amostragem $52(2 \mathrm{~cm} \leq D A P \leq 5 \mathrm{~cm})$

$$
2.857
$$

Sub-amostragem $\mathrm{S} 3$ (DAP $<2 \mathrm{~cm}$ )

$116.000 \quad \$ 61.250 \quad \$ 45.500$


com uma única espécie sendo que metade delas apresentaram também um único indivíduo - estas famílias são as mais ameaçadas requerendo atenção redobrada num programa de conservação.

Eschweilera coriacea (matamatá branco) e Lecythis idatimon (jatereua) são as espécies de maior destaque na florística do fragmento tanto na abundância quanto no IVI.

A dinâmica da riqueza e da abundância apresentou crescimento de ambas as variáveis no período considerado.

Independentemente da classe diamétrica, verificou-se o egresso de 56 espécies face ao ingresso de outras 68 , gerando um ganho líquido de 12 espécies*ano ${ }^{-1}$.

A dinâmica da riqueza da regeneração natural é muito intensa: houve um egresso de $36,7 \%$ de espécies e $2,2 \%$ de famílias em contraposição a um ingresso de $72,8 \%$ e $30,4 \%$, respectivamente; já a dinâmica da abundância é mais intensa que a da florística: $54,2 \%$ de mortalidade em oposição a $93,1 \%$ de recrutamento.

Mais da metade do total das espécies registradas na regeneração natural 'entraram' na amostra após a primeira medição. Também, a nível de família, observou-se, no período de um ano, a saída de apenas uma familia em contraposição ao ingresso de outras 14 , elevando a riqueza em quase um terço do total,

A maior mortalidade absoluta anual foi verificada em Bauhinia cf rutilans e Mabea aff speciosa (300 e
21 indivíduos, respectivamente).

Rinorea negleta e Lecythis idatimon foram as espécies que mais recrutaram, em termos absolutos: 171 e 89 espécimes, respectivamente.

A razão recrutamento/mortalidade foi em quaisquer das classes diamétricas, sempre superior a unidade ou, em outras palavras, o número de indivíduos mortos é sempre inferior ao número de indivíduos recrutados.

A média de mais de 138.000 indivíduos ${ }^{*} \mathrm{a}^{-1}$ da regeneração natural corresponde a mais de $97 \%$ do total estimado para todas as classes diamétricas que foi de 142.820 indivíduos* $\mathrm{ha}^{-1}$.

A proporção para se obter uma árvore, uma arvoreta e uma vara é de, respectivamente 297,160 e 48 mudas do estoque bruto.

Tanto a área basal, quanto o volume de madeira e a biomassa apresentaram crescimento no período.

Em resumo: a riqueza e a abundância vegetal aumentaram no periodo, assim como a área basal e a biomassa.

\section{Agradecimentos}

Às instituições e aos funcionários do Fundo Nacional do Meio Ambiente - FNMA/MMA e do Conselho Nacional de Desenvolvimento Científico e Tecnológico - CNPq/MCT, expressamos nossos sinceros agradecimentos.

\section{Bibliografia citada}

Balee, W. 1986. Análise preliminar de inventário florestal e etnobotânica Ka'apor (Maranhão). Bol. Mus. Para. Emilio Goeldi, sér. Bot., 2(2):141-67.

Bastos, A. M. 1948. As matas de Santa Maria 
do Vila Nova, Território do Amapá. An. Bras. de Economia Florestal, 1:121-8.

Black, G. A.; Dobzansky, T.; Pavan, C. 1950. Some attenpts no estimate species diversity and population density of trees in Amazonian Forest. Bot. Geog, 111:41325.

Brown, I. F.; Nepstad, D.C.; Pires, I.O.; Luz, L. M.; Alechandrea, A.S. 1992. Carbon storage and Land-use in Extractive Reserves, Acre, Brazil. Environmental Conservation, 19(4):307-15.

Brown, S; Giullespic, A.J.R.; Lugo, A. 1989. Biomass estimation methods for tropical forests with applications to forest inventory data. Forest Science, 35(4):881-902.

Cain, S.A.; Castro, G.M.A.; Pires, J.M.; Silva, N.T. 1956. Application of some phytosociological techniques to brasilian rain forest. An. J. Bot.: 43:911-41.

Campbell, D.G.; Daly, D.C.; Prance, G.T.; Maciel, U.M. 1986. Quantitative ecological inventory of terra firme and várzea tropical forest on the Rio Xingu, brasilian Amazon. Brittonia, 38 (4):369-93.

Carey, E.V.; Brown, S.; Giullespic, A.J.R.; Lugo, A.E. 1994. Tree mortality in mature lowland tropical moist and tropical lower montane moist forests of Venezuela. Biotropica, 26(3):255-65.

Carvalho, J.O.P. de. 1999. Dinâmica de florestas naturais e sus importância para o manejo florestal. $I n$ : Simpósio Silvicultura na Amazônia Oriental: Contribuiçòes do Projeto Embrapa/ DFID, Belém, Resumos Expandidos, Embrapa-DFID. p.174-9.

Carvalho, J.O.P. de; Lopes, J. do M.A.; Silva, J.N.M. 1999. Dinâmica da diversidade de espécies em uma floresta de terra firme na Amazônia brasileira relacionada à intensidade de exploração. In: Simpósio Silvicultura na Amazônia Oriental: Contribuições do Projeto Embrapa/ DFID, Belém, Resumos Expandidos, EmbrapaDFID. p.167-73.

Clinebell II, R.R.; Phillips, O.L.; Gentry, A.H.; Stark, N.; Zuuring, H. 1995. Prediction of neotropical tree and liana species richeness from soil and climatic data. Biodiversity and Conservation, 4:56-90.
Condit, R.; Hubbcll, S.P.; Lafrankie, J.V.; Sukumar, R.; Mankaran, M.; Foster, R.B.; Ashton, P. 1996. Specics-area and speciesindividual relation ships for tropical tress: a composion of three 50 ha plots. $J$. Ecol. 84: $549-62$.

Cottam, G.; Curtis, J. T. 1956. The use of distance mensures in phytosociological sampling. Ecology., 37(3):45I-60.

Dantas, M.; Muller, N.R.M. 1980. Estudos fitoecológicos do trópico úmido brasileiro 1 - Aspectos fitosociológicos de mata de terra roxa na região de Altamira. In: Congresso Nacional de Botânica, 30. São Paulo. Anais ... p. $205-218$.

Dantas, M.; Rodrigues, J.A.; Muller, N.R.M. 1980. Estudos fito-ecológicos do trópico úmido brasileiro: aspectos fítosociológicos de mata sobre latossolo em Capitão Poço, PA. Bol. Pesq. Embrapa/Cpatu, Belém, 9:1-19.

Diniz, T.D.A.S. 1986. Caracterizaçào climática da Amazônia oriental. In: Embrapa/Cpatu-Gtz. Belém. 3:13

Ducke, A.; Black, G. A. 1954. Notas sobre a fitogeografia da Amazônia braileira. $B o l$. Téc. do Inst. Agron. Norte, Belém, 29:1-48.

Felfili, J.M. 1995. Diversity, structure and dynamics of a gallery forest in central Brazil. Vegetatio, 117:1-15.

IBDF/SUDAM. 1988. Levantamento da alteração da cobertura vegetal natural do Estado do Pará. Convênio IBDF/ SUDAM - Progrma de monitoramento da cobertura florestal do Brasil Belém. Relatório Técnico.

Jardim, F.C.S. 1990. Mortalidade e crescimento na floresta equatorial de terra firme. $\mathrm{Bol}$. Mus. Para. Emilio Goeldi, sér: Bot. 6(2):227-34.

Lisboa, P.L.B.; Lisboa, R.C.L. 1984. Inventário florestal em Rondônia. I - Rodovia Presidente Médici - Costa Marques, Km 90. In: Congresso Nacional de Botânica, 35. Manaus. Anais . . .

Magurran, A.E. 1988. Ecological diversity and its measturement. Princeton Univ. Press. New Jcrsey. 420 p.

Margalef, R. 1968. Perspectives in Ecological Theory. Chicago, University of Chicago 
Press, 112 pp.

Margalef, R. 1972. Homage to Evelyn Hutchinson or why is there an upper lilit to diversity. Trans. Connect. Acad. Arts Sci., 44:221-35.

Moraes, V.H.F. 1970. Periodicidade de crescimento do tronco em árvores da floresta amazônica. Pesq. agropec. bras. 5:315-20.

Mueller-Dombois, D.; Ellemberg, H. 1974. Aims and Methods of vegetation ecology. New York, John Willey and Sous (ed.). p. 45-135.

Oliveira, A.A. 1997. Diversidade, estrutura e dinámica do componente arbóreo de uma floresta de terra firme de Manaus, Amazonas. São Paulo: Instituto de Biocièncias, USP, 155 p. Tese Doutorado.

Phillips, O.L.; Gentry, A.H. 1994. Increasing turnover through time in tropical forests. Science, 263: 954-8.

Phillips, O.L.; Hall, P; Gentry, A.H.; Sawyer, S.A.; Vasquez, R. 1994. Dinamics and species richness of tropical rain forests. Proc. Natl.Acad. Sci. USA, 91:2805-9.

Pires, J.M.; Dobzansky, T,; Black, G.A. 1953. An estimate of the number of species of trees in an Amazonian Forest Community, Bot. Gaz., 114(4): 467-77.

Pires, J.M.; Moraes, V.H.F. 1966. Composição da mata de terra firme da Reserva Mocambo. Belém: Instituto de Pesquisa e Experimentação Agropecuária do Norte. Relatório Anual. 57p.

Pires, J.M.P. 1976. Aspectos ecológicos da floresta amazónica. In: Congresso Brasileiro de Florestas Tropicais, 2, Mossoró (RN). Anais ... Coleção Mossoroense v. LXV, p. 235-87.

Porto, M.L.; Longhi, H.M.; Citadini, V.; Ramos, R.F.; Mariath, J.E.A. 1976. Levantamento fitosociológico em área de "mata-de-baixio" na Estação Experimental de Silvicultura Tropical, INPA, Manaus. Acta Amazonica, 6(3): 301-18.

Prance, G.T.; Rodrigues, W.A.; Silva, M.F. 1976. Inventário florestal de um hectare de mata de terra firme, $\mathrm{Km} 30$ da estrada Manaus - Itacoatiara. Acta Amazonica, $6(1) ; 9-35$.
Putz, F.R.; Milton, K. 1982 Tree mortaly rate on Barro Colorado Island. In: Leigh Jr, E.G.; A.S.; Windsor, D.M. (eds). The ecology of a tropical forest, Smithsonian Institution Press, Washington DC. p.95-100.

Rankin-De Merona, J.M.; Hutchings, R.W.; Lovejoy, T.E. 1990.Tree mortality and recruitment over a five-year period in the undisturbed upland rainforest of the Central Amazon. In: Gentry, A.H. (ed). Four Neotropical Forests. Yale Univ. Press., New Haven, CT. p. 573-84.

Rankin-De Merona, J.M.; Prance, G.T.; Hutchings, R.W.; Silva, M.F.; Rodrigues, W.A.; Uehling, M.E. 1992. Preliminar results of a large-escale tree inventory of upland rain forest in the Central Amazon. Acta Amazonica, 22(4): 493-534.

Ricklefs, R. E. 1979. Ecology. 2a ed. New York, Chiron Press. p. 688-7.

Rodrigues, W.A. 1963. Estudo de 2,6ha de mata de terra firme da Serra do navio, Território do Amapá. Bol. Mus. Para. Emilio Goeldi, sér: Bot., 19:1-44.

Salomão, R.P.; Lisboa, P.L.B. 1988. Análise ecológica da vegetação de uma floresta pluvial tropical de terra firme, Rondônia. Bol. Mus. Para. Emilio Goeldi, sér. Bot., 4(2):195-233.

Salomão, R.P.; Santos, J.U.M. 1997. A floresta tropical primária densa de Porto Trombetas, Pará. In: Congresso Nacional de Botânica, 48, Croto. Resumos ..... p.245-6.

Salomão, R.P. 1991. Uso de parcelas permanentes para estudos de vegetação florestal I. Municipio de Marabá, Pará. Bol. Mus. Para. Emilio Goeldi, sér: Bot., 7(2): 543-604.

Salomão, R.P.; Rosa, N.A.; Matos, A. 2000. Estudo e monitoramento da floresta tropical primária visando a restauração da paisagem florestal em áreas degradadas da Amazônia brasileira. In: Simpósio Nacional de Recuperaçào de Areas Degradadas, 4, Curitiba. SOBRADE/ FURBE (Anais/Resumos - CD-ROM).

Salomão, R.P.; Silva, M.F.F.; Rosa, N.A. 1988. Inventário ecológico em floresta pluvial tropical de terra firme, Serra Norte, 
Carajás, Pará. Bol. Mus. Para. Emilio Goeldi, sér. Bot.. 4(1):1-46.

Shannon, C.E.; Wiener, W. 1949. The Mathematical Theory of Communication. Urbana. University of Illinois Press, $117 \mathrm{pp}$.

Silva, M.F.F.; Rosa, N.A.; Salomão, R.P. 1986. Estudos botânicos na área do Projeto Carajás. 3 - Aspectos florísticos da mata do aeroporto de Serra Norte - PA. Bol. Mus. Para. Emilio Goeldi, sér Bot., 2(2): 169-87.

Simpson, E.H. 1949. Measurement of diversity. Nature, 163:688.

Sorensen. T 1943. A method of establishing groups of equal amplitude in plant sociology based on similarityof species contents. Kong. Danske Vidensk. Selsk. Biol. Skr. 5(4): 1-34.

Uhl, C. 1982. Tree dynamics in a species rich tierra firme forest in Amazonia, Venezuela. Acta Ci. Venez., 33:72-7.

Uhl, C.; Buschbacher, R.; Serrão, E.A.S. 1988. Abandoned pastures in lastern Amazonia. I. Patterns of plant sucession, Journal of Ecology, 76:663-81.

Veillon, J.P.; Konrad, V.W.; Garcia, N. 1976. Estudio de la masa forestal y su dinamismo en parcelas de diferentes tipos ecológicos de bosques naturales de las tierras bajas venezolanas.Rev. For: Venez., 19(26): 73-106.

Aceito para publicação em 18/07/2002 
Anexo 1. Dinâmica da abundância e da diversidade florística nas três unidades amostrais permanentes da floresta tropical primária densa de Município de Peixe-Boi, Pará.

\begin{tabular}{|c|c|c|c|c|c|c|c|c|c|c|c|c|}
\hline \multirow[t]{2}{*}{ ESPÉCIE } & \multicolumn{2}{|c|}{$\mathrm{AP}$} & \multicolumn{2}{|c|}{ S1 } & \multicolumn{2}{|c|}{$\$ 2$} & \multicolumn{2}{|c|}{ \$3 } & \multicolumn{2}{|c|}{ TOTAL } & \multicolumn{2}{|c|}{$\Delta 98 \rightarrow 99^{4}$} \\
\hline & ANO 1 & ANO 2 & ANO 1 & ANO 2 & ANO 1 & ANO 2 & ANO 1 & ANO 2 & ANOI & ANO 2 & & \\
\hline 1. Abarema cochleatum & 1 & 1 & & & & & & & 1 & 1 & & k \\
\hline 2. Abuta grandiflora & & & & & & & & 2 & & 2 & & $1-2$ \\
\hline 3. Abuta sp & & & & 1 & & & & & & 1 & & {$[-2$} \\
\hline 4. Acacia multipinnata & 5 & 7 & 4 & 2 & & 1 & & 3 & 9 & 13 & +4 & $\uparrow$ \\
\hline 5. Acacia panicusata & & & & & 1 & & & & 1 & & & $E-2$ \\
\hline 6. Acanthacea indel. $53-34-30$ & & & & & & & & 1 & & 1 & & $1-2$ \\
\hline 7. Ajouea sp & 1 & 1 & & & & & & & 1 & 1 & & K \\
\hline 8. Allophylus cl. punctatus & 1 & 1 & & & & & & & 1 & 1 & & k \\
\hline 9. Amajoua corymbosa & & & & & 1 & 1 & & & 1 & 1 & & K \\
\hline 10. Amaioua guianensis & 2 & 2 & & & & & & & 2 & 2 & & $\mathrm{~K}$ \\
\hline 11. Amaioua sp & & & & & & & 2 & & 2 & & & $E-2$ \\
\hline 12. Amajoa ct. guianensis & & & & & & & & 4 & & 4 & & $1-2$ \\
\hline 13. Ambelania acida & 2 & 1 & 2 & 2 & 1 & 1 & & & 5 & 4 & -1 & $\downarrow$ \\
\hline 14. Ampetocera edentuta & 10 & 10 & 2 & 4 & 3 & 3 & & 5 & 15 & 22 & +7 & $\uparrow$ \\
\hline 15. Amphirrox of. surinamensis & 1 & 1 & 4 & 4 & 25 & 26 & & 1 & 30 & 32 & +2 & $\uparrow$ \\
\hline 16. Anacardium giganteum & 1 & 1 & & & & & & & 1 & 1 & & K \\
\hline 17. Andira retusa & 1 & 1 & 1 & 1 & & & & & 2 & 2 & & k \\
\hline 18. Aniba guianensis & & & & & & & & 4 & & 4 & & $1-2$ \\
\hline 19. Aniba panurensis & 1 & 1 & & & & & & & 1 & 1 & & K \\
\hline 20. Anmona sp & 1 & 1 & & & & & & & 1 & 1 & & K \\
\hline 21. Aparisthmium cordatum & 3 & 2 & 2 & & & & 1 & 1 & 6 & 3 & +3 & $\uparrow$ \\
\hline 22. Apeiba burchelfit & 25 & 25 & 2 & 1 & 1 & 2 & & & 28 & 28 & & K \\
\hline 23. Apeiba echinata & 5 & 5 & & & & & & & 5 & 5 & & K \\
\hline 24. Apendiculata sp & & & & & & & 1 & & 1 & & & $E-2$ \\
\hline 25. Apuleia leiocarpa & 9 & 9 & & & & & . & & 9 & 9 & & K \\
\hline 26. Aracea indet. $\$ 3.08 .43$ & & & & & & & & 2 & & 2 & & $1-2$ \\
\hline 27. Arrabidaea cinamonea & & & & & & & 7 & & 7 & & & $E-2$ \\
\hline 28. Arrabidaea nigrescens & &. & & & & & & 6 & & 6 & & $1-2$ \\
\hline 29. Arrabidaea sp & & & & & & & & $\mathfrak{t}$ & & 1 & & $1-2$ \\
\hline 30. Arrabidaea fuberculata & 1 & 1 & 1 & 1 & & & 2 & & 4 & 2 & -2 & $\downarrow$ \\
\hline 31. Aspidosperma desmanthum & 8 & 8 & & & 1 & 1 & & & 9 & 9 & & K \\
\hline 32. Aspidosperma oteanum & 1 & 1 & & & & & 3 & 4 & 4 & 5 & -1 & $\downarrow$ \\
\hline 33. Aspidosperma nitidum & 1 & 1 & & & & & & & 1 & 1 & & K \\
\hline 34. Asplenium sp & & & & & & & & 1 & & 1 & & $i-2$ \\
\hline 35. Astrocaryum gynacantum & & & 3 & 4 & & & & 1 & 3 & 5 & +2 & $\uparrow$ \\
\hline 36. Astronium lecointei & 5 & 5 & & & & & & 1 & 5 & 6 & +1 & $\uparrow$ \\
\hline 37. Bachitris ct. gracite & & & & & & & & 1 & & 1 & & $1-2$ \\
\hline 38. Bagassa gujanensis & 1 & 1 & & & & & & & 1 & 1 & & $k$ \\
\hline 39. Banara guianensis & & & $\uparrow$ & 1 & & & & & 1 & 1 & & K \\
\hline 40. Bauthinia of. rutitans & 4 & 11 & 5 & 7 & 12 & 8 & 352 & 47 & 373 & 73 & -300 & $\downarrow$ \\
\hline 41. Bauhinia cuprionites & & & & & & & & 77 & & 77 & & $1-2$ \\
\hline 42. Bauhinia guianensis & 5 & 6 & 8 & 9 & 1 & & 11 & 2 & 25 & 17 & -8 & $\downarrow$ \\
\hline 43. Bauthinia jariensis & 2 & 2 & 2 & 2 & & & & & 4 & 4 & & K \\
\hline 44. Bauhinia macrostachya & 3 & 3 & & & & & & & 3 & 3 & & K \\
\hline 45. Bauhinia viridiffora & & & 1 & 1 & 3 & 3 & & & 4 & 4 & & $\mathrm{~K}$ \\
\hline 46. Bertiera guianensis & & & & & 2 & & & & 2 & & & $\mathrm{E}-2$ \\
\hline 47. Brosimum guianensis & & & & & 2 & 2 & & & 2 & 2 & & $\mathrm{~K}$ \\
\hline 48. Brosimum tactescens & 1 & 1 & 1 & 1 & 3 & 3 & & & 5 & 5 & & $\mathrm{~K}$ \\
\hline 49. Brosimum rubescens & 2 & 2 & & & & & & 1 & 2 & 3 & +1 & $\uparrow$ \\
\hline 50. Buchenavia sp & & $y$ & & & & & & & & 1 & & $1-2$ \\
\hline
\end{tabular}


Anexo 1. Continuação

\begin{tabular}{|c|c|c|c|c|c|c|c|c|c|c|c|c|}
\hline \multirow[t]{2}{*}{$\overline{\text { ESPÉCIE }}$} & \multicolumn{2}{|c|}{ AP } & \multicolumn{2}{|c|}{ s1 } & \multicolumn{2}{|c|}{ S2 } & \multicolumn{2}{|c|}{$\$ 3$} & \multicolumn{2}{|c|}{ TOTAL } & \multicolumn{2}{|c|}{$\triangle 9 B \rightarrow 99^{c}$} \\
\hline & ANO 1 & $\mathrm{ANO} 2$ & ANO 1 & ANO 2 & ANO 1 & ANO 2 & ANO 1 & ANO 2 & ANOr & ANO 2 & & \\
\hline 51. Bunforrestia candolleana & & & & & & & 1 & 7 & 1 & 7 & +6 & $\uparrow$ \\
\hline 52. Calathea cf, zingibelifera & & & & & & & & 2 & & 2 & & $\mathrm{I}-2$ \\
\hline 53. Catathea sp & & & & & & & 51 & 135 & 51 & 135 & +84 & $\uparrow$ \\
\hline 54. Calycolpus sp & 4 & 4 & & & & & & & 4 & 4 & & K \\
\hline 55. Calyptranthes ct. macrophylta & & & 7 & 8 & 3 & 3 & & & 10 & 11 & +1 & $\uparrow$ \\
\hline 56. Calyptranthes sp & 5 & 5 & 4 & 4 & & & & & 9 & 9 & & $\mathrm{~K}$ \\
\hline 57. Campomanesia cf. aromatica & 2 & 2 & & & & & & & 2 & 2 & & K \\
\hline 58. Carapa guianensis & 4 & 4 & & & & & & & 4 & 4 & & $\mathrm{~K}$ \\
\hline 59. Caryocar giabrum & 4 & 4 & & & 1 & 1 & & & 5 & 5 & & $\mathrm{~K}$ \\
\hline 60. Caryocar vilossum & 1 & 1 & & & & & & & $i$ & 1 & & K \\
\hline 61. Casearia decandra & 1 & 1 & & & & & & & 1 & 1 & & K \\
\hline 62. Casearia mariquitensis & 5 & 4 & & & & & & & 5 & 4 & .1 & $\downarrow$ \\
\hline 63. Cassipourea gutanensis & & & 1 & 2 & & & & & 1 & 2 & +1 & $\uparrow$ \\
\hline 64. Cecropia distachya & 19 & 14 & & & & & & & 19 & 14 & -5 & $\downarrow$ \\
\hline 65. Cecropia obtusa & 1 & 1 & & & & & & & 1 & 1 & & k \\
\hline 66. Cecropia sciadophylla & 2 & 2 & & & 1 & 1 & & & 3 & 3 & & K \\
\hline 67. Chamaecrista bahiae & 1 & 1 & 1 & 1 & & & & & 2 & 2 & & $\mathrm{k}$ \\
\hline 68. Chanouchiton kapleri & 1 & 1 & & & & & 1 & 1 & 2 & 2 & & K \\
\hline 69. Cheiloclinium cognatum & & & & & & & & 1 & & 1 & & $1-2$ \\
\hline 70. Clatisia ifficifolia & & & 2 & 1 & & & 1 & 2 & 3 & 3 & & K \\
\hline 71. Clarisia racemosa & 7 & 8 & & & & & & 2 & 7 & 10 & +3 & $\hat{\imath}$ \\
\hline 72. Coccoloba parinensis & & & 1 & 2 & 5 & 5 & 1 & & 7 & 7 & & K \\
\hline 73. Coccoloba $s p$ & & & & & & & & 7 & & 7 & & $1-2$ \\
\hline 74. Combretum laxum & 1 & 1 & 3 & 3 & 5 & 5 & 1 & & 10 & 9 & -1 & $\downarrow$ \\
\hline 75. Combretum rotumdifotium & & & 4 & 4 & & & & & 4 & 4 & & $\mathrm{~K}$ \\
\hline 76. Conceveiba guianensis & 3 & 3 & 1 & & & & & & 4 & 3 & -1 & $\downarrow$ \\
\hline 77. Copaitera duckei & 1 & 1 & & & & & & & 1 & 1 & & $\mathrm{~K}$ \\
\hline 78. Cordia goatdiana & 2 & 2 & & & & & & & 2 & 2 & & $\mathrm{~K}$ \\
\hline 79. Cordia scabrida & 5 & 6 & & & & & & & 5 & 6 & +1 & $\uparrow$ \\
\hline 80. Cordia scabritolia & 2 & 2 & & & & & & & 2 & 2 & & K \\
\hline 81. Costus tasius & & & & & & & 3 & 13 & 3 & 13 & +10 & $\uparrow$ \\
\hline 82. Courepia aft. magnotiaetolia & 1 & 1 & & & & & & & 1 & 1 & & K \\
\hline 83. Couepia guianensis & 9 & 8 & 1 & 1 & 1 & 1 & 3 & 2 & 14 & 12 & 2 & $\downarrow$ \\
\hline 84. Couratari gujanensis & 2 & 2 & 2 & 2 & & & & & 4 & 4 & & K \\
\hline 85. Couratari multifora & 5 & 5 & & & & & & & 5 & 5 & & K \\
\hline 86. Coussarea ovalis & 7 & 7 & & 1 & & & 2 & 2 & 9 & 10 & +1 & $\uparrow$ \\
\hline 87. Coussarea paniculata & 4 & 4 & & & & & & & 4 & 4 & & k \\
\hline 88. Croton cajuçara & 1 & 1 & & & & & & & 1 & 1 & & K \\
\hline 89. Croton matourense & 3 & 3 & & & & & & & 3 & 3 & & $\mathrm{x}$ \\
\hline 90. Cupania scrobicutata & 2 & 2 & 3 & 4 & 6 & 5 & & & 11 & 11 & & k \\
\hline 91. Curarea cf toxicofera & & & & & & & 1 & & 1 & & & $E-2$ \\
\hline 92. Cybiarithus sp & & & & & & & 7 & & 7 & & & $E-2$ \\
\hline 93. Dalbergia subcymosa & & & 1 & 1 & & & & & 1 & 1 & & K \\
\hline 94. Davilta aff. Kunthit & 2 & 3 & & 1 & 3 & 3 & & & 5 & 7 & +2 & $\uparrow$ \\
\hline 95. Dendrobangia boliviana & 9 & 9 & 1 & 1 & & & & & 10 & 10 & & K \\
\hline 96. Denis floribunda & & & & & 2 & 1 & & & 2 & 1 & .1 & $\downarrow$ \\
\hline 97. Demis tatifolia & 1 & 1 & & & & & & & 1 & $t$ & & k \\
\hline 98. Derris sp & $=$ & & & & & 1 & & & & 1 & & $\mathrm{I}-2$ \\
\hline 99. Diatium guianense & 5 & 6 & 3 & 3 & & & & & 8 & 9 & +1 & $\uparrow$ \\
\hline 100. Dichapetalum sp & & & & 1 & & & & 2 & & 3 & & $1-2$ \\
\hline 101. Dichapetaum rugosum & & & & & & & & 1 & & 1 & & $1-2$ \\
\hline 102. Diospyrus artanthaefolia & 1 & 1 & & & & & & & 1 & 1 & & K \\
\hline 103. Diospyrus melinonit & 6 & 7 & 2 & 2 & 1 & 1 & & 2 & 9 & 12 & +3 & $\uparrow$ \\
\hline 104. Dipteryx odorata & 1 & 1 & 1 & 1 & 1 & 1 & 1 & 3 & 4 & 6 & +2 & $\uparrow$ \\
\hline 105. Dotiocarpus att, major & & & & & & & 3 & & 3 & & & $\varepsilon-$ \\
\hline 106. Dotiocampus cl. brevipedicalla & & & & & & & & 5 & & 5 & & $1-2$ \\
\hline
\end{tabular}


Anexo 1. Continuação

\begin{tabular}{|c|c|c|c|c|c|c|c|c|c|c|c|c|}
\hline \multirow{2}{*}{ ESPECIE } & \multicolumn{2}{|c|}{ AP } & \multicolumn{2}{|c|}{$\$ 1$} & \multicolumn{2}{|c|}{$\$ 2$} & \multicolumn{2}{|c|}{$\$ 3$} & \multicolumn{2}{|c|}{ TOTAL, } & \multicolumn{2}{|c|}{$\Delta 98 \rightarrow 994$} \\
\hline & ANO 1 & $\overline{\text { ANO } 2}$ & ANO 1 & $\overline{A N O} 2$ & ANO 1 & $\overline{A N O} 2$ & ANO 1 & ANO 2 & ANO1 & ANO2 & & \\
\hline 107. Doliocampus dentatus & & & & & 2 & 2 & 1 & & 3 & 2 & -1 & $\downarrow$ \\
\hline 108. Doliocarpus gracilis & & & & & & & & 2 & & 2 & & $t-2$ \\
\hline 109. Doliocarpus magium & & & & & & & & 2 & & 2 & & $i-2$ \\
\hline 110. Drypetes variabilis & 2 & 2 & & & & & & & 2 & 2 & & K \\
\hline 111. Duguetia flagelaris & & & & & 1 & 1 & 7 & 17 & 8 & 18 & +10 & $\uparrow$ \\
\hline 112. Enterolobium schomburgkii & 2 & 2 & & & & & & & 2 & 2 & & K \\
\hline 113. Episcia sp & & & & & & & & $g$ & & $\vartheta$ & & $1-2$ \\
\hline 114. Erisma uncinatum & 8 & B & & & 2 & 4 & 8 & 17 & 18 & 29 & +11 & $\uparrow$ \\
\hline 115. Eschweilera aft ovata & 8 & B & & & & & & & 8 & 8 & & K \\
\hline 116. Eschweitera amazonica & 1 & 1 & & & & & & & 1 & 1 & & $\mathrm{k}$ \\
\hline 117. Eschueilera apiculata & & 1 & & & & & & & & 1 & & $1-2$ \\
\hline 118. Eschueitera cf. collina & 9 & 9 & & & & 1 & & & 9 & 10 & +1 & $\uparrow$ \\
\hline 119. Eschweilera coniacea & 120 & 120 & 8 & 10 & 11 & 10 & 17 & 22 & 156 & 162 & +6 & $\uparrow$ \\
\hline 120. Eschneilera pedicallata & 9 & 9 & 4 & 5 & 2 & 1 & & & 15 & 15 & & K \\
\hline 121. Estenochiaena marginata & & & & & & & 1 & & 1 & & & $E-2$ \\
\hline 122. Eugenia ct brachypoda & & & 1 & & & 1 & & & 1 & 1 & & $k$ \\
\hline 123. Eugenia cf. coffegefolia & & & 1 & 1 & 1 & 1 & & & 2 & 2 & & K \\
\hline 124. Eugenia cf. teijoi & 4 & 4 & & & & & & & 4 & 4 & & K \\
\hline 125. Eugenia cf. tambertia & 4 & 4 & & & . & & & & 4 & 4 & & K \\
\hline 126. Eugenia flavescens & & & & & & & & 1 & & 1 & & $1-2$ \\
\hline 127. Eugenia mimits & 1 & 1 & & & & & & & 1 & 1 & & K \\
\hline 128. Eugenia pamisit & 3 & 2 & 3 & 3 & 1 & 2 & 1 & 2 & $B$ & 9 & +1 & $\uparrow$ \\
\hline 129. Eugenia schomburgkit & & & 1 & 1 & 1 & 1 & & & 2 & 2 & & k \\
\hline 130. Eugenia sp & & & & & & & 1 & & 1 & & & $\mathrm{I}:-2$ \\
\hline 191, Eugenia spt & 1 & 1 & & & & & & & 1 & 1 & & K \\
\hline 132. Eugenia sp2 & 3 & 3 & & & 1 & 1 & & & 4 & 4 & & K \\
\hline 133. Eugenia spos & 1 & 1 & & & & & & & 1 & 1 & & k \\
\hline 134. Eugenia tapacumensis & & & 1 & & 2 & 1 & & & 3 & 1 & -2 & $\downarrow$ \\
\hline 135. Euterpe oleracsa & & & & & & & & 1 & & 1 & & $1-2$ \\
\hline 136. Faranea $s p$ & & & & & & & & 1 & & 1 & & $1-2$ \\
\hline 137. Ficus gameleira & 1 & 1 & & & & & & & 1 & 1 & & K \\
\hline 138. Forsteronia guionansis & & 1 & 1 & 1 & 3 & 4 & 3 & 2 & 7 & $\theta$ & 41 & $\uparrow$ \\
\hline 139. Franchetelila anibifolia & 6 & 5 & 2 & 2 & 1 & 1 & & & 9 & 8 & -1 & $\downarrow$ \\
\hline 140. Franchetefla cl. reticutata & & & 1 & 1 & 1 & 1 & & & 2 & 2 & & K \\
\hline 141. Franchelella gongnpii & 4 & 4 & & & 1 & 1 & 1 & & 6 & 5 & $\cdot 1$ & $\downarrow$ \\
\hline 142. Franchetella jariensis & 12 & 12 & & & & 1 & & & 12 & 13 & +1 & $\dagger$ \\
\hline 143. Franchetelia reticulata & & & & & & & & 2 & & 2 & & $1-2$ \\
\hline 144. Franchetelfa sagotiana & 8 & 9 & 3 & 3 & 4 & 3 & & & 15 & 15 & & $\mathrm{~K}$ \\
\hline 145. Fusaea fongifolia & & 1 & 2 & $i$ & & & & 2 & 2 & 4 & +2 & $\uparrow$ \\
\hline 146. Geissospermum sericeum & 7 & 7 & 8 & 9 & 3 & 1 & & & 18 & 17 & $\cdot 1$ & $\downarrow$ \\
\hline 147. Geissospermum velozii & 7 & 8 & & & & & & & 7 & 8 & +1 & $\uparrow$ \\
\hline 148. Geophila sp & & & & & & & & 16 & & 16 & & $1-2$ \\
\hline 149. Geophitla gracitis & & & & & & & 2 & & 2 & & & $F,-2$ \\
\hline 150. Geophilla sp & & . & & & & & & 1 & & 1 & & $1-2$ \\
\hline 151. Goupia glabra & 8 & 8 & & & & & & & 8 & 8 & & $\mathrm{k}$ \\
\hline 152. Guarea kunthians & 5 & 5 & & & 2 & 2 & & & 7 & 7 & & $\mathrm{~K}$ \\
\hline 153. Guarea kunthii & & & & & & & 5 & & 5 & & & $1-2$ \\
\hline 154. Guatteria cl. paeppigiana & 3 & 3 & & & 1 & 1 & 1 & 1 & 5 & 5 & & $\mathbf{K}$ \\
\hline 155. Gurania cf. acuminata & & & & & & 1 & & & & 1 & & $1-2$ \\
\hline 156. Gurania SP & & & & & & & & 1 & & 1 & & $i-2$ \\
\hline 157. Gustavia augusta & 27 & 29 & 38 & 38 & 10 & 12 & 1 & & 76 & 79 & +3 & $\uparrow$ \\
\hline 158. Hebepotalum humirifolium & 1 & 1 & & & & & & & 1 & 1 & & K \\
\hline 159. Heisteria acuminata & 5 & 5 & 6 & 6 & 4 & 5 & 1 & 1 & 16 & 17 & +1 & $\uparrow$ \\
\hline 160. Heisteria scandens & & & & & 3 & 3 & 1 & 1 & 4 & 4 & & K \\
\hline 161. Heliconia acuminata & & & & & & & & 4 & & 4 & & $1-2$ \\
\hline 162. Helicostylis ct. elegans & 1 & 1 & & & & & & & 1 & 1 & & k \\
\hline 163. Helicostylis pedunculata & 1 & 1 & & & 2 & 2 & & 1 & 3 & 4 & +1 & $\uparrow$ \\
\hline
\end{tabular}


Anexo 1. Continuação

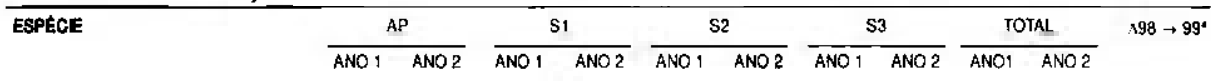

164. Helicostylis scabra

165. Helicostylis $s p$

166. Helicostylis tomentosa

22

167. Hernandia gujanensis

168. Hoterapleris jenmanii

169. Heieropteris $s p$

170. Himatanthus sucuuba

171. Mippocratea of. ovata

172. Hippocratea sp

173. Hippocrateacea indet.

174. Hiraea sp

175. Hinelila racemosa

176. Huminanthera duckei

177. Huminanthera $s p$

178. Humiriastrum aff. excelsum

179. Hymenaea parvifotia

180. Hymenolobium excelsum

181. Ichnanthus panicoides

182. Hex parvilora

183. indet. $51 \cdot 01 \cdot 15$

184. inder. $51.01-50$

185. Indet. S2.01.008

186. Indet: \$2-05-052

187. Indet. S2-31-190

188. indet. 53-0r-11

189. inga alba

190. inga aunisteilae

191. Inga cayennensis

192. Inga ef. bourgoni

193. inga ct. microcalyx

194. inga falcistipusa

195. inga gracilitotia

196. inga heterophylla

197. inga macrophylla

198. inga marginata

199. Inga nibida

200. inga rubiginosa

201. inga sp

202. Inga splandens

203. inga stiputaris

204. Inga thibaudiana

205. triattera exomhiza

206. Hyanihera sagotiana

207. ischnosophon gracilis

208. Jacaranda copaia

209. Jacaratia spinosa

210. Lacmellea aculeata

211. Lacmeltea floribunda

212. Lacunaria crenata

213. Lacunaria jenmanii

$\begin{array}{cc}1 & 1 \\ 10 & 9 \\ & \\ 1 & 1 \\ 5 & 5 \\ 1 & 1\end{array}$

$+2$


Anexo 1. Continuação

\begin{tabular}{|c|c|c|c|c|c|c|c|c|c|c|c|c|}
\hline \multirow[t]{2}{*}{ ESP'ECE } & \multicolumn{2}{|c|}{ AP } & \multicolumn{2}{|c|}{ S1 } & \multicolumn{2}{|c|}{$\$ 2$} & \multicolumn{2}{|c|}{ \$3 } & \multicolumn{2}{|c|}{ TOTAL } & \multicolumn{2}{|c|}{$198 \times 99^{\circ}$} \\
\hline & ANO 1 & ANO 2 & ANO 1 & ANO 2 & ANO 1 & ANO 2 & ANO 1 & ANO 2 & ANO1 & ANO 2 & & 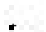 \\
\hline 221. Licania heteromorpha & 9 & 9 & 1 & 1 & & & & & 10 & 10 & & K \\
\hline 222. Licania kunthiana & & & 1 & 1 & & & & & 1 & 1 & & K \\
\hline 223. Llcanja membranacea & 2 & 2 & 1 & 1 & & & & & 3 & 3 & & K \\
\hline 224. Licania octandra & 3 & 3 & & & & & & & 3 & 3 & & K \\
\hline 225. Licania sp & 1 & 1 & & & & & & & 1 & 1 & & K \\
\hline 226. Licaria armeniaca & & & 1 & 1 & & & & & 1 & 1 & & $\mathrm{~K}$ \\
\hline 227. Lindackeria paraensis & 2 & 2 & & & & & & & 2 & 2 & & K \\
\hline 228. Luheopsis duckeana & 3 & 3 & & & & & & & 3 & 3 & & K \\
\hline 229. Mabea aft. speciosa & 39 & 37 & 2 & 1 & 2 & 3 & 31 & 12 & 74 & 53 & $\cdot 21$ & $\downarrow$ \\
\hline 230. Macfadyena unguis-cali & 1 & 1 & & & 1 & & & & 2 & 1 & -1 & $\downarrow$ \\
\hline 231. Machaerium ferox & 1 & 2 & & & & 1 & & & 1 & 3 & +2 & $\uparrow$ \\
\hline 232. Machaerium madeirensis & & & & & & & 1 & & 1 & & & $E-2$ \\
\hline 233. Mạchaerium sp & 2 & 2 & & & & & & 1 & 2 & 3 & +1 & $\uparrow$ \\
\hline 234. Macherrium ct. inundatum & & & & & & & 3 & & 3 & & & $\mathrm{E}-2$ \\
\hline 235. Malpighiacea indel. S3-15-15 & & & & & & & 3 & & 3 & & & $E-2$ \\
\hline 236. Matpighiacea indet. $\$ 3 \cdot 25 \cdot 12$ & & & & & & & 7 & & 7 & & & $E-2$ \\
\hline 237. Manaosefta cf. cordifolia & & & & & & & & 2 & & 2 & & {$[-2$} \\
\hline 238. Manaosella corditolia & & & & & & & & 2 & & 2 & & $\mathrm{I}-2$ \\
\hline 239. Manihot ct. brachyloba & & & 1 & 1 & & & & & 1 & 1 & & K \\
\hline 240. Mansoa of. angustidens & & & & & & & & 4 & & 4 & & $3-2$ \\
\hline 241. Mapouria ct. fockeana & & & 2 & 2 & 2 & 1 & & & 4 & 3 & -1 & $\downarrow$ \\
\hline 242. Mapouria sp & 2 & 2 & & & & & & & 2 & 2 & & K \\
\hline 243. Maprounea gutanensis & 2 & 2 & & & & & & & 2 & 2 & & $\mathrm{~K}$ \\
\hline 244. Maquira coriacea & & & & & 1 & 1 & & & 1 & $t$ & & $\mathrm{~K}$ \\
\hline 245. Maquira guianensis & 7 & 7 & 1 & 1 & 3 & 2 & 2 & 1 & 13 & 11 & $\cdot 2$ & $\downarrow$ \\
\hline 246. Marcgravia polyantha & & & 1 & 1 & & & & & 1 & 1 & & K \\
\hline 247. Maripa sp & 1 & 1 & & & & & & & 1 & 1 & & K \\
\hline 248. Mammaroxytum racemosum & 10 & 10 & & & & & & & 10 & 10 & & K \\
\hline 249. Matayba guianensis & 3 & 3 & & & & & & & 3 & 3 & & K \\
\hline 250. Matayba macrostylis & 12 & 11 & & & & & & & 12 & 11 & -1 & $\downarrow$ \\
\hline 251. Maytenus cf. ebenitolia & & & & & 1 & & & & 1 & & & F-2 \\
\hline 252. Maytenus myrsinoides & 11 & 11 & & & & & & & 11 & 11 & & K \\
\hline 253. Maytenus sp & 1 & 1 & & & & & & & 1 & 1 & & $\mathrm{~K}$ \\
\hline 254. Memora alamandiffora & & & & & 10 & 10 & 1 & 3 & 11 & 13 & -2 & $\downarrow$ \\
\hline 255. Memora bracteosa & & & & & & & 1 & & 1 & & & E-2 \\
\hline 256. Memora flavida & & & & & 24 & 23 & 4 & 19 & 28 & 42 & +14 & $\uparrow$ \\
\hline 257. Memora magnifica & & & & & 3 & 3 & 2 & 4 & 5 & 7 & +2 & $\uparrow$ \\
\hline 258. Memora schomburgkii & & & & & & & & 3 & & 3 & & $\mathrm{I}-2$ \\
\hline 259. Metrodorea flavida & 2 & 2 & & & & & & & 2 & 2 & & K \\
\hline 260. Miconia ceramicama & & & & & & & 26 & 23 & 26 & 23 & -3 & $\downarrow$ \\
\hline 261. Miconia ct. ruficalyx & & & & & & & & 1 & & 1 & & $1-2$ \\
\hline 262. Miconia of. serialis & 2 & 2 & & & & & & & 2 & 2 & & K \\
\hline 263. Miconia ct. stenostachya & & & 1 & & & & & & 1 & & & E-2 \\
\hline 264. Miconia cf. telrasperma & 3 & 3 & & 1 & 2 & 1 & & & 5 & 5 & & K \\
\hline 265. Miconia elata & & & & & & & & 4 & & 4 & & $1-2$ \\
\hline 266. Miconia nervosa & & & & & 3 & 4 & & & 3 & 4 & +1 & $\uparrow$ \\
\hline 267. Miconia poeppigii & & & 2 & 2 & 1 & 1 & & & 3 & 3 & & K \\
\hline 268. Miconia sp & & & & & & & 1 & & 1 & & & Li-2 \\
\hline 269. Miconia spy & 1 & 1 & & & & & & & 1 & 1 & & K \\
\hline 270. Micropholis guianensis & 9 & 8 & 1 & 1 & 3 & 3 & 2 & 1 & 15 & 13 & -2 & $\downarrow$ \\
\hline 271. Micropholis venulosa & 2 & 2 & & & 1 & 1 & & & 3 & 3 & & K \\
\hline 272. Minquartia guianensis & 5 & 4 & & & & & & & 5 & 4 & -1 & $\downarrow$ \\
\hline 273. Monotagma cf spicatum & & & & & & & 12 & & 12 & & & $E-2$ \\
\hline 274. Monotagma cf. pturispicatum & & & & & & & & $\theta$ & & 8 & & $I-2$ \\
\hline 275. Monotagma sp & & & & & & & & 2 & & 2 & & $1-2$ \\
\hline 276. Monstera obliqua & & & & & & & 12 & & 12 & & & $E-2$ \\
\hline 277. Moracea indet $53-0 \mathrm{r}-01$ & & & & & & & 3 & & 3 & & & $E-2$ \\
\hline 278.Moracea indet $\$ 3-08-01$ & & & & & & & 3 & & 3 & & & $E-2$ \\
\hline
\end{tabular}


Anexo 1. Continuação

ESPECE

279. Moracea indet. S3-09-06

280. Mourisi brachyanthera

28t. Mourir calocarpa

282. Mouriri sagotiana

283. Moutabea guianensis

284. Mussatia sp

285. Myrcia amazonica

286. Myrcia bracleata

287. Myrcia ch. lanuotteana

288. Myrcia tallax

289. Myrcia sylvatica

290. Myreiania Aloribunda

291. Myrciaria tenella

292. Nectandra aff. gjobosa

293. Neciandra cuspidata

294. Neer sp

295. NeBs spi

296. Noea SP2

297. Neisonia Sp

298. Neoplychocarpus apodanthus

299. Neoxythece cladantha

300. Newtonia psilostachya

301. Neweonia suaveotens

302. Nyclaginacea indet. $\$ 3.01-05$

303. Ocolea cajumari

304. Ocotea caudata

305. Ocotea rubra

306. Ocolea sp

307. Cenocarpus distichus

308. Otyra tatifolia

309. Ondontadenia sp

310. Orchidacea indet. 53-01-48

3it. Ormosia flava

312. Ormosia nobilis

313. Omosia paraensis

314. Omosia sp

315. Parahancomia amapa

316. Pariana $s p$

317. Parinari rodolphii

318. Parkia gigantocarpa

319. Parkia penduta

320. Parkia utei

321. Paulinia $s p$

322. Pedata stotzsch

323. Pedata sp

324. Philodendron aff. obliqua

325. Philodendion ct. micranthum

326. Phitodendfon of. muricatum

327. Phitodendron squamiterum

328. Picramnia $s p$

329. Piper cameconnectivum

330. Piper ottonoides

33r. Piper piresii

332. Piper schwackei

333. Piper sp?

334. Piper sp2

335. Pisonia sp

$$
\frac{\text { AP }}{\text { ANO1 ANO2 }} \frac{S 1}{\text { ANO! ANO2 }} \frac{\text { S2 }}{\text { ANO1 ANO2 }} \frac{S 3}{\text { ANO1 ANO2 }} \frac{\text { TOTAL }}{\text { ANO1 ANO2 }} \quad \Delta 98 \rightarrow 99^{4}
$$$$
2 \quad 2 \quad \mathrm{E}-2
$$

$\begin{array}{lllllllllll}14 & 14 & 1 & 1 & 4 & 4 & 1 & 3 & 20 & 22 & +2\end{array}$

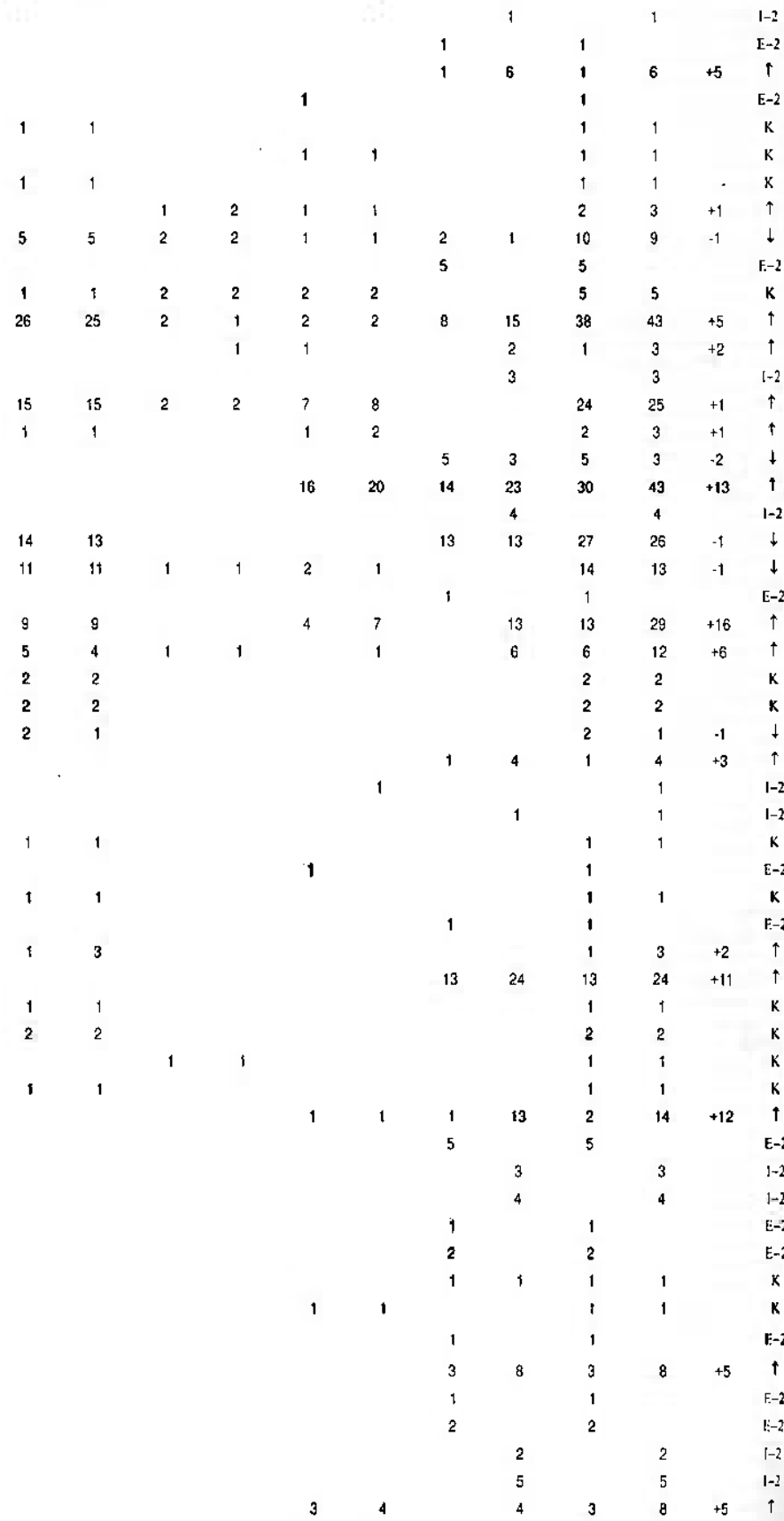


Anexo 1. Continuação

\begin{tabular}{|c|c|c|c|c|c|c|c|c|c|c|c|c|}
\hline \multirow[t]{2}{*}{ ESPÉCIE } & \multicolumn{2}{|c|}{$\mathrm{AP}$} & \multicolumn{2}{|c|}{ S1 } & \multicolumn{2}{|c|}{ \$2 } & \multicolumn{2}{|c|}{$\underline{\mathrm{S3}}$} & \multicolumn{2}{|c|}{ TOTAL } & A98 $\rightarrow$ & 994 \\
\hline & ANO 1 & ANO 2 & ANO 1 & ANO 2 & ANO 1 & ANO 2 & ANO 1 & ANO 2 & ANO1 & ANO 2 & & \\
\hline 336. Planchonetia oblanceolata & 7 & 7 & 2 & 2 & & & & 1 & 9 & 10 & +1 & $\hat{\imath}$ \\
\hline 337. Planchonella prieurif & 3 & 4 & & & & & & & 3 & 4 & +1 & $\uparrow$ \\
\hline 338. Pogonophora schomburgkiana & 10 & 10 & 1 & 1 & & & & & 11 & 11 & & $\mathrm{~K}$ \\
\hline 339. Poraqueiba guianensis & 29 & 28 & 2 & 2 & 3 & 4 & 1 & 1 & 34 & 35 & +1 & $\uparrow$ \\
\hline 340. Pourouma acuminata & 1 & 1 & 2 & 1 & 1 & 1 & & & 4 & 3 & .1 & $\downarrow$ \\
\hline 341. Pourouma guianensis & 1 & 1 & & & & & & & 1 & 1 & & $\mathrm{~K}$ \\
\hline 342. Pourouma $s p$ & 4 & 4 & & & & & & & 4 & 4 & & $\mathrm{k}$ \\
\hline 343. Pourouma velutina & 3 & 4 & & & & & & 8 & 3 & 12 & +9 & $\uparrow$ \\
\hline 3\$4. Pouteria echinocarpa & 1 & 1 & & & & & & & 1 & 1 & & $x$ \\
\hline 345. Pouteria guianensis & 1 & 1 & & & & & & & 1 & 1 & & K \\
\hline 346. Pouteria heterosepata & 1 & 1 & & & & & & & 1 & 1 & & k \\
\hline 347. Pouteria hispida & 2 & 1 & & & 1 & 1 & & & 3 & 2 & -1 & $\downarrow$ \\
\hline 348. Pouteria fasiocarpa & 4 & 4 & 2 & 2 & & & & 2 & 6 & 8 & +2 & $\uparrow$ \\
\hline 349. Pouteria oblanceolata & 1 & 1 & & & & & & & 1 & 1 & & k \\
\hline 350. Pouteria spt & 1 & 1 & & & & & & & 1 & 1 & & K \\
\hline 351. Pouteria $5 \rho 2$ & 1 & 1 & & & & & & & 1 & 1 & & $\mathrm{k}$ \\
\hline 352. Pradosia granulosa & 2 & 3 & & & & & & & 2 & 3 & +1 & $\uparrow$ \\
\hline 353. Prionostemma angulala & & & & & & & & 1 & & 1 & & $1-2$ \\
\hline 354. Prionostemma aspera & & & 3 & 2 & 3 & 1 & 2 & 1 & 8 & 4 & 4 & $\downarrow$ \\
\hline 355. Protium alf. subserratum & 5 & 5 & & & 1 & 1 & & & 6 & 6 & & $\mathbf{k}$ \\
\hline 356. Protium decandrum & 8 & 8 & 2 & 2 & 3 & 3 & & & 13 & 13 & & k \\
\hline 357. Protitum guianensis & & & & & 1 & 1 & & & 1 & 1 & & K \\
\hline 358. Prottum heptaphyflum & & & 3 & 3 & 2 & 1 & & & 5 & 4 & -1 & $\downarrow$ \\
\hline 359. Protium pallidum & 1 & 1 & & & & & & & 1 & 1 & & $\mathrm{k}$ \\
\hline 360. Protium pilosum & 3 & 2 & 5 & 6 & 18 & 15 & 24 & 29 & 50 & 52 & +2 & $\uparrow$ \\
\hline 361. Protium sagotianum & 15 & 16 & & & 1 & 1 & & 22 & 16 & 39 & +23 & $\uparrow$ \\
\hline 362. Profium sp & & & & & & & 1 & & 1 & & & E-2 \\
\hline 363. Protium sp 1 & 1 & 1 & & & & & & & 1 & 1 & & K \\
\hline 364. Protium sp2 & 1 & 1 & & & & & & & $\mathbf{I}$ & 1 & & K \\
\hline 365. Prolium sp3 & 1 & 1 & & & & & & & 1 & 1 & & $\kappa$ \\
\hline 366. Protium spruceanum & & & 1 & 1 & 2 & 2 & & & 3 & 3 & & K \\
\hline 367. Protium tenuifotium & 11 & 11 & 1 & 2 & 1 & 1 & & & 13 & 14 & +1 & $\uparrow$ \\
\hline 368. Protium tritolioialum & 8 & 11 & 8 & 8 & 2 & 2 & & 2 & 18 & 23 & +5 & $\uparrow$ \\
\hline 369. Prunus myntifotia & 3 & 2 & & & & & & & 3 & 2 & +1 & $\uparrow$ \\
\hline 370. Pseudima frutescens & & & & & 3 & 2 & & & 3 & 2 & +1 & $\uparrow$ \\
\hline 371. Psoudoconnarus ct. macropty/lus & & & & & & & & 1 & & 1 & & $1-2$ \\
\hline 372. Psevoolmedia laevigata & & & & & & & & 2 & & 2 & & $1-2$ \\
\hline 373. Pseudoimedia murure & 2 & 2 & 1 & 1 & & & 1 & 1 & 4 & 4 & & K \\
\hline 374. Psychotria appendiculata & & & & & & & 2 & & 2 & & & $\mathrm{E}-2$ \\
\hline 375. Psychatria sp & & & & & & & & 4 & & 4 & & $1-2$ \\
\hline 376. Plerocanpus rohrii & 3 & 3 & & & & & & & 3 & 3 & & K \\
\hline 377. Oualea albibora & 2 & 2 & & & 1 & 1 & & & 3 & 3 & & K \\
\hline 378. Quatea paraensis & 8 & 8 & & & & 1 & & & $\mathrm{~B}$ & 9 & +1 & $\uparrow$ \\
\hline 379. Outisna cl. duckei & 3 & 3 & & & & & & & 3 & 3 & & $x$ \\
\hline 380. Quitina obovata & & & & & & & 1 & & 1 & & & E-2 \\
\hline 381. Quïna paraensis & & & & & & & & 1 & & 1 & & $1-2$ \\
\hline 382. Radikolerefla macrocarpa & 3 & 3 & & & & & & & 3 & 3 & & K \\
\hline 383. Rhedia acuminata & & & & & & & & 4 & & 4 & & $1-2$ \\
\hline 384. Rheedia acuminata & 3 & 3 & & & & & & & 3 & 3 & & $\mathrm{~K}$ \\
\hline 385. Finorea negiecta & & & 2 & 2 & 46 & 49 & 46 & 214 & 94 & 265 & +171 & $\uparrow$ \\
\hline 386. Finorea passoura & 1 & 1 & 1 & 1 & 3 & 3 & & 8 & 5 & 13 & +8 & $\uparrow$ \\
\hline 387. Sacoggiontis guianensis & 1 & 1 & & & & & & & 1 & 1 & & K \\
\hline 388. Salacia ct impressilofia & & & & & & & 1 & 5 & 1 & 5 & 44 & $\uparrow$ \\
\hline 389. Salacia ef. mantiniana & & & 2 & 2 & & & & & 2 & 2 & & K \\
\hline 390. Salacia impressifola & & & & & 1 & & & & 1 & & & E-? \\
\hline 391. Salacia sp & & 1 & & & & & & & & 1 & & $1-2$ \\
\hline 392. Sandwithiodoxa egregia & 9 & 11 & 3 & 3 & 4 & 3 & 1 & 6 & 17 & 23 & +6 & $\hat{\imath}$ \\
\hline 393. Sapiurit aft. pueppigiti & 7 & 7 & & & & & & 1 & 7 & 8 & +1 & $\uparrow$ \\
\hline 394. Sapium mamieri & 1 & 1 & & & & & & & 1 & 1 & & K \\
\hline 395. Scheffiera morototoni & 6 & 6 & & & & & & & 6 & 6 & & $\mathrm{k}$ \\
\hline 396. Schyzophon geacilis & & & & & & & & 1 & & 1 & & $1-2$ \\
\hline 397. Sciadoienia ef sprucei & & & & & & & 1 & & 1 & & & $E-2$ \\
\hline 398. Sclemalobium paraense & 9 & 9 & & 1 & 2 & 1 & & 1 & $\$ 1$ & 12 & +1 & $\uparrow$ \\
\hline 399. Senna bahiai & 1 & 1 & & & & & & & 1 & 1 & & $\mathbf{K}$ \\
\hline 400. Serjania membranacea & & & & & 1 & 2 & 6 & 2 & 7 & 4 & -3 & $\downarrow$ \\
\hline 401. Senania cf. grandifolia & & & 1 & & & & & & 1 & & & $E-2$ \\
\hline 402. Serjania cf. membranacea & & & & & 1 & 2 & 6 & 2 & 7 & 4 & -3 & $\downarrow$ \\
\hline 403. Simaruba amara & 8 & 8 & & & 1 & 1 & & 2 & 9 & 11 & +2 & $\uparrow$ \\
\hline 404. Simanuba ct. multito & 1 & 1 & & & & & & & 1 & 1 & & $k$ \\
\hline 405. Sloanea garckeana & 6 & 6 & & & 1 & 1 & & & 7 & 7 & & K \\
\hline 406. Stoanea guianensis & & 1 & & & & & & & & 1 & & $1-2$ \\
\hline 407. Sioanes parvitora & & & 2 & 2 & & & & & 2 & 2 & & $\mathrm{k}$ \\
\hline
\end{tabular}


Anexo 1. Continuação

\begin{tabular}{|c|c|c|c|c|c|c|c|c|c|c|c|c|}
\hline \multirow[t]{2}{*}{ ESPECIE } & \multicolumn{2}{|c|}{$\mathrm{AP}$} & \multicolumn{2}{|c|}{$\mathrm{S1}$} & \multicolumn{2}{|c|}{$\mathrm{S} 2$} & \multicolumn{2}{|c|}{$\mathrm{s3}$} & \multicolumn{2}{|c|}{ TOTAL } & \multirow{2}{*}{\multicolumn{2}{|c|}{$498 \rightarrow 99^{4}$}} \\
\hline & ANO 1 & ANO 2 & ANO 1 & ANO 2 & ANO 1 & $\mathrm{ANO} 2$ & ANO 1 & ANO 2 & ANO1 & ANO 2 & & \\
\hline 408. Siganea sp & 1 & 1 & & . & & & & 2 & 1 & 3 & +2 & $\uparrow$ \\
\hline 409. Sloanea spt & & & 1 & & & & & & 1 & & & L:-2 \\
\hline 410. Smitax sp & & & & & & & & 3 & & 3 & & {$[-2$} \\
\hline 411. Solanum satvijfolia & & & & & 2 & 2 & & & 2 & 2 & & K \\
\hline 412. Soracea muricutata & & & & & 1 & 1 & 1 & & 2 & 1 & -1 & $\downarrow$ \\
\hline 413. Sparathanthelium tupiniquinorum & & & 1 & 1 & 1 & 1 & & & 2 & 2 & & $\kappa$ \\
\hline 414. Sterculia pilosa & 1 & 1 & & & & & & & 1 & 1 & & $k$ \\
\hline 415. Stercutia pruriens & $B$ & 10 & 11 & 10 & 3 & 4 & & 2 & 22 & 26 & +4 & $\uparrow$ \\
\hline 416. Stercutia speciosa & 2 & 2 & & & & & & & 2 & 2 & & K \\
\hline 417. Stigmaphylton so & & & & & 1 & 1 & & & 1 & 1 & & K \\
\hline 418. Strychnos cf. hirsuta & & & & & 1 & 1 & 2 & & 3 & 1 & -2 & $\downarrow$ \\
\hline 419. Strychnos ct. pechii & 1 & 1 & 1 & 1 & 3 & 2 & & & 5 & 4 & -1 & $\downarrow$ \\
\hline 420. Strychnos sp & & & & & & 1 & & 2 & & 3 & & $1-2$ \\
\hline 421. Stryphnodendron pulcherrimum & 1 & 1 & & & & & & & 1 & 1 & & $k$ \\
\hline 422. Styzophytlum riparium & & & & 1 & 1 & & 2 & 3 & 3 & 4 & +1 & $\uparrow$ \\
\hline 423. Suertzia arborescens & 1 & 1 & & & & & & & 1 & 1 & & K \\
\hline 424. Suartzia brachyrhachis & & & 2 & 2 & & & & & 2 & 2 & & k \\
\hline 425. Symphonia globutifera & 18 & 19 & 2 & 1 & 2 & 2 & B & 11 & 30 & 33 & +3 & $\uparrow$ \\
\hline 426. Tabebuia serratifotia & 4 & 5 & 2 & 1 & 2 & 2 & 2 & 3 & 10 & 11 & +1 & $\uparrow$ \\
\hline 427. Tabernaemontana angutata & & & & & & & 1 & 3 & 1 & 3 & +2 & $\uparrow$ \\
\hline 428. Tabemaemontana macrocalyx & & & & 1 & 1 & 2 & 1 & & 2 & 3 & +1 & $\uparrow$ \\
\hline 429. Tachigatia alba & 7 & 7 & & & & & & & 7 & 7 & & K \\
\hline 430. Tachigatia myfmecophila & 9 & 11 & 2 & 2 & 3 & 2 & & & 14 & 15 & +1 & $\uparrow$ \\
\hline 431. Tachigatia panicutala & 3 & 3 & 4 & 4 & 1 & 2 & & & 8 & 9 & +1 & $\uparrow$ \\
\hline 432. Tatisia cf. Tongitolia & & & & & 2 & 2 & 1 & & 3 & 2 & $\cdot 1$ & $\downarrow$ \\
\hline 433. Tatisia of microphytla & & & $z$ & 2 & 5 & 5 & & & 7 & 7 & & $\mathrm{~K}$ \\
\hline 434. Talisia floribunda & & & 1 & 1 & & & & & 1 & 1 & & K \\
\hline 435. Talisia macrophyfla & & & & & 1 & 1 & 1 & & 2 & 1 & -1 & $\downarrow$ \\
\hline 436. Tatisia mollis & & & & & 2 & 2 & & & 2 & 2 & & K \\
\hline 437. Talisia retusa & 6 & 7 & 4 & 4 & 3 & 3 & & & 13 & 14 & +1 & $\uparrow$ \\
\hline 438. Talisia sp & 1 & 1 & & & & & & 3 & 1 & 4 & +3 & $\hat{i}$ \\
\hline 439. Talista subatbens & 1 & 1 & & & & & & & 1 & 1 & & K \\
\hline 440. Tapirira guianensis & 16 & 15 & & & 1 & 1 & & & 17 & 16 & -1 & $\downarrow$ \\
\hline 441. Terminalia amazonica & 3 & 3 & & & & & & & 3 & 3 & & K \\
\hline 442. Terminalia argentea & 2 & 1 & & & & & & & 2 & 1 & -1 & $\downarrow$ \\
\hline 443. Teminalia guianensis & & & 1 & & & & & & 1 & & & $\mathrm{E}-2$ \\
\hline 444. Terminalia sp & 1 & 1 & & & & & & & 1 & 1 & & k \\
\hline 445. Tetracera cf. volubitis & & & 1 & 1 & & & & & 1 & 1 & & K \\
\hline 446. Tetracera sp & & & & & & & 2 & & 2 & & & $\leftleftarrows-2$ \\
\hline 447. Tetracera willdenowiana & & & 2 & 2 & 3 & 3 & & & 5 & 5 & & $\mathrm{~K}$ \\
\hline 448. Telragastris altissima & 3 & 3 & & & & & & & 3 & 3 & & K \\
\hline 449. Tetragastris panamensis & & & & & 1 & 1 & & & 1 & 1 & & $k$ \\
\hline 450. Tetrapleryx of discolor & 1 & 1 & 1 & 1 & 3 & 3 & & & 5 & 5 & & K \\
\hline 451. Theobroma grandifforum & 1 & 1 & & & & & & & 1 & 1 & & $\mathrm{~K}$ \\
\hline 452. Theobroma speciosum & 7 & 7 & 5 & 6 & 5 & 5 & 2 & & 19 & 18 & -1 & $\downarrow$ \\
\hline 453. Thyrsodium paraense & 11 & 11 & 2 & 2 & & & & 1 & 13 & 14 & +1 & $\uparrow$ \\
\hline 454. Tovomita aft. schomburgkil & 2 & 2 & & & & & & & 2 & 2 & & $\mathrm{~K}$ \\
\hline 455. Tovomita choysiana & & & & & & & & 2 & & 2 & & $1-2$ \\
\hline 456. Trattinickia moifolia & 4 & 4 & & & & & & & 4 & 4 & & $k$ \\
\hline 457. Trichifia of. quadrijuga & & & 2 & 2 & 1 & 2 & & & 3 & 4 & +1 & $\uparrow$ \\
\hline 458. Trichitia of. rubra & 2 & 2 & & & & & & & 2 & 2 & & $k$ \\
\hline 459. Trichitla micrantha & 12 & 12 & 4 & 4 & 5 & 4 & 7 & 2 & 28 & 22 & -6 & $\downarrow$ \\
\hline 460. Trichiffia schombungkii & 12 & 13 & 3 & 2 & 1 & 3 & $i$ & 9 & 17 & 27 & -10 & $\downarrow$ \\
\hline 461. Trichilfa septentionalis & 5 & 5 & 1 & 1 & 1 & 1 & 1 & & 8 & 7 & -1 & $\downarrow$ \\
\hline 462. Trichifia sp & & & & & & & & 3 & & 3 & & $1-2$ \\
\hline 463. Trymatococcus of. ofigandius & 1 & 1 & & 1 & & & & & 1 & 2 & +1 & $\uparrow$ \\
\hline 464. Virota michetii & 25 & 27 & 7 & 6 & 6 & 5 & 5 & 6 & 43 & 44 & +1 & $\uparrow$ \\
\hline 465. Virola surinamensis & & & & & & & 1 & 2 & 1 & 2 & +1 & $\uparrow$ \\
\hline 466. Vismia cayennensis & 1 & 1 & & & & & & & 1 & 1 & & $\mathrm{k}$ \\
\hline 467. Vitex triftora & 1 & 1 & $t$ & 1 & & & & 1 & 2 & 3 & +1 & $\uparrow$ \\
\hline 468. Vochysia visniaefotia & 6 & 6 & 1 & 1 & & & 7 & & 14 & 7 & .7 & $\downarrow$ \\
\hline 469. Xylopia nitida & & 1 & & & & & & & & 1 & & $\mathrm{H}$ \\
\hline 470. Zanthoxylum regneffiana & 4 & 4 & & & & & & & 4 & 4 & & $k$ \\
\hline 471. Zolfernia paraensis & 1 & 1 & 1 & & & & & & 2 & 1 & $\cdot 1$ & $\downarrow$ \\
\hline NO TORAL DE ESPECIES & 250 & 258 & 132 & 132 & 142 & 142 & 121 & 150 & 396 & 406 & - & - \\
\hline Ne TOTAL HDNIDUOS & 1.385 & 1.417 & 343 & 347 & 412 & 411 & 882 & 1.075 & 3.022 & 3.250 & - & - \\
\hline
\end{tabular}

${ }^{4}$ Nota: $A P=D A P \geq 10,0 \mathrm{~cm} ; S 1=10,0 \mathrm{~cm}>D A P \geq 5,0 \mathrm{~cm} ; \mathrm{S} 2=5,0 \mathrm{~cm}>D A P \geq 2,0 \mathrm{~cm} ; \mathrm{S} 3=D A P<2,0 \mathrm{~cm}$;. $\Delta_{98} \rightarrow{ }_{99}$ traduz a variação corrente anual do $\mathrm{n}^{\circ}$ de individuos das espécies que os apresentaram 2em ambos os anos; quando se refere à espécie informa $\mathrm{I}-2$ = espécie que ingressou na amostra no Ano 2 e $\mathrm{E} \cdot 2$ = espécie egressa (que saiu) da amostra no Ano 2. 